\title{
Effect of a Gurney flap on the performance of an axial fan
}

\author{
Chen Liu, Haijun Xie, Jun Xu, Chen Jian*, Ren Dai \\ School of Energy and Power Engineering, University of Shanghai for Science and Technology \\ E-mail:09900589r@connect.polyu.hk
}

\section{Abstract:}

The Gurney flap (GF) is a miniature lift-enhancement device and is usually mounted at the trailing

edge of an airfoil. The GF and has been successfully applied to isolated airfoils, multi-element

airfoils, and aircraft wings as well as helicopter rotor due to its attractive features of simplicity, cost-

effective and separation control. The GF also has aroused the attention of researchers in the turbomachinery industry. However, limited studies are currently available on the application of a

GF to an axial fan.

Hence, in this paper, we conduct a wind tunnel and computational fluid dynamic (CFD) investigation on an axial fan profiled with a NACA 65-(12)10 airfoil to evaluate the effect of the GF on the performance of the fan. We also present the detailed flow features of the fan with and without the GF after validating the simulation results with the experimental results. The experimental results show that as the GF is installed higher on the fan blade, it can produce a higher total pressure rise accompanied with a greater loss of efficiency. The installation of the GF also enlarges the work capacity of the fan. Detailed flow field analysis, including the surface pressure distribution, vorticity distribution at the trailing edge and streamline distribution of the fan, is carried out to understand the mechanisms of the effect of the GF on the performance of the fan.

Keywords: Axial fan; Gurney flaps; wind tunnel testing; CFD investigation; pressure rise coefficient; 


\section{2 \\ 1 Introduction}

The Gurney flap (GF), invented by a race car driver Dan Gurney in 1960, is a small lift-enhancement device and is usually mounted at the trailing edge of an airfoil. A Gurney flap with a height of $2 \%$ of the airfoil chord $(2 \% \mathrm{C})$ can increase the maximum lift coefficient by $21 \%$ and lift-to-drag ratio by $35 \%$ on a NACA0012 airfoil [1]. The lift enhancement mechanism stated by Liebeck is that the existence of the Gurney flap generates a pair of counter-rotating vortices downstream of the Gurney flap and creates a region of low pressure. This region contributes to the positive liftelement distribution on the upper suction side of the airfoil and negative lift-element distribution on the lower pressure surface of the airfoil.

Because of its structural simplicity, ease of manufacture and cost-effectiveness [2], the GF has attracted much attention from researchers in many areas. Most of the previous researchers have focused on the utilization of a Gurney flap on isolated airfoils, multi-element airfoils and aircraft wings.

Wang J., et al[3] studied the influence of the mounting location of the GF on the performance of a NACA0012 airfoil. Four different mounting locations (S = 0\%C, 2\%C, 4\%C and 6\%C) were studied. The results showed that the increment of the lift coefficient decreased when the GF was moved forward to the leading edge. The mounting angle was also studied by Traub L.W., et al[4]. They found that a perpendicular GF generated the lowest lift-to-drag ratio. GFs at $45^{\circ}$ and $60^{\circ}$ produced the maximum lift-to-drag ratio. Kobayashi T., et al[5] came to a similar conclusion. The configuration of the GF was investigated by Dan and Vijgen, P.M., et al [6, 7] using a sawtoothed GF for an isolated airfoil. They indicated that the sawtoothed GF improved the lift coefficient and 
43

reduced the drag coefficient. This was attributed to a reduction in the effective windward area.

A large number of researchers have used GFs on dual or multi-element airfoils or wings for aircraft applications. Storms B.L, et al[8, 9] utilized a $0.5 \%$ chord GF and a $1 \%$ chord GF on a NACA632215(B) airfoil to increase the maximum lift coefficient by $10.3 \%$ when the deflection angle of the slotted flap was 42 degrees. Myose R et al[10, 11]mounted a 1\% chord GF at the trailing edge of the main and flap elements on a two-element GA(W)-2 airfoil. It was found that the "Flap GF" and "Flap main GF" can enhance the lift coefficient, but the "main GF" reduces the lift coefficient compared to a "clean" airfoil. An investigation of the effect of a GF on multi-element airfoils can also be found in references [12]. Traub, L. W, et al[13]performed a wind tunnel investigation to examine the dependence of the Gurney flap on various geometric descriptive parameters, and the data suggested that the lift augmentation of the Gurney flap varied linearly with the flap height, porosity and projected height of the flap normal to the surface. Li et al[14] tested four traditional plain GFs with heights ranging from $0.01 \mathrm{C}$ to $0.05 \mathrm{C}$ as well as four $0.05 \mathrm{C}$ serrated GFs with different serration heights ranging from $0.01 \mathrm{C}$ to $0.05 \mathrm{C}$ on a cropped nonslender delta wing. They found that a proper GF can provide a significant improvement in performance. Zhan and Ji Wang[15]also tested a delta wing with GFs and apex flaps. The experimental results indicated that the lift coefficient was improved for different angles of attack (AOA) for a delta wing with GFs and apex flaps. Albertani, $R$ [16] used a simple GF to improve the aerodynamic characteristics of a micro aerial vehicle wing. With GFs, the maximum lift coefficient was increased by $26.5 \%$ in comparison with clean wings. Additional applications of the GF on wings can be found in $[17,18]$. 
appealing features of the simplicity, lift enhancement and separation control of the GF have also given rise to the attention of experts in other fields, especially in the turbomachinery industry. Janus, J. M.,[19]conducted numerical and experimental analyses of the size effect of the GF on the performance of a cascade configuration derived from a single rotating fan with six blades. Two baseline geometries with varied GFs were tested; one had a sharp trailing edge, and the other had a round trailing edge. The results suggested that the flap size should be limited to between $1 \% \sim 1.5 \%$ of the chord. Byerley, A. R., et al[20] experimentally investigated the influence of the Gurney flap on the location and size of the laminar separation bubble on turbine blades in a linear cascade. The testing data showed that the lift generated by the blade was increased and that the Gurney flap also produced a larger wake. It was suggested that Gurney flaps might possibly be implemented in a semi-passive manner. They could be deployed for low a Reynolds-number operation and then retracted at high Reynolds numbers when separation is not present. Large GFs with heights of 0.1C, $0.2 \mathrm{C}$ and $0.3 \mathrm{C}[21]$ were tested at a fan facility. It was found that blades with GFs produce higher pressures than those without GFs. A GF of $30 \%$ of the chord created large volumetric flowrates and reduced the sound pressure level by $4 \mathrm{~dB}$. A GF of $10 \%$ of the chord increased the static efficiency by $18 \%$. The effect of GFs on the performance of a centrifugal fan was investigated by Thomas, M. K. D et al [22] at low Reynolds numbers. The height of the GFs was $3.175 \mathrm{~mm}$, which was $15.9 \%$ of the blade exit height. They observed that the GF improved the performance of the fan considerably at low Reynolds numbers and marginally at higher Reynolds number.

As indicated by the above-mentioned literature survey, there have been a large number of studies on the effect of GFs on one-element airfoils, multi-element airfoils and wings. However, there have 
been few studies of the application of GFs to fans, especially with regard to axial fans. The application of GFs seems to be a promising approach to improve the performance of axial fans.

Thus, the purpose of this paper is to investigate the performance of an axial fan with GFs by experimental measurement. Flow features were interrogated by the CFD (Computational Fluid Dynamics) code to comprehensively understand the physics involved in the GF. The pressure coefficient, total pressure efficiency and static pressure efficiency of the clean fan and axial fans with varied plate GFs were tested first. Then, the simulation results were compared with the tested results to validate the computational strategies. In the end, we analyzed and visualized the flow features of a fan with GFs. The latter work offers a theoretical foundation for further optimization of axial fans.

\section{Experimental set up}

\subsection{Testing Facility}

Axial fan testing was performed in the Energy and Power Engineering Department of the University of Shanghai for Science and Technology. The testing rig shown in Figure 1 was built in compliance with the Chinese national standard for fan measurement (GB/T1236-2000). The flow quality of the plenum is guaranteed. Beyond a velocity of $10 \mathrm{~m} / \mathrm{s}$, the variation in the total pressure and static pressure field is less than $0.1 \%$ and $0.3 \%$, respectively, while the maximum deflection of the flow angle is below \pm 0.2 degree, and the turbulence intensity is less than $0.5 \%$ at the test section.

The flow plenum is divided into four parts (long entrance duct, diffusion nozzle, plenum and exhaust duct). The lengths of these four parts are approximately $3580 \mathrm{~mm}, 587 \mathrm{~mm}, 1600 \mathrm{~mm}$ and 
105 106

$370 \mathrm{~mm}$, respectively. The diameter of the long duct is approximately $498 \mathrm{~mm}$, and the diameter of the inlet chamber is approximately $1240 \mathrm{~mm}$. Upstream of the long entrance duct, there is a flow nozzle, where several additional taped holes are mounted. Circumferential tapes are used to measure the static pressure difference across the flow nozzle to calculate the volumetric flowrate $q_{m}$. A flow regulating valve (position 3) is installed $2145 \mathrm{~mm}$ downstream of the plenum inlet to adjust the volumetric flowrate of incoming air. An auxiliary fan (position 4) is located $985 \mathrm{~mm}$ downstream of the regulating valve to adjust the flow rate through a frequency converter to provide sufficient pressure at the desired airflow and overcome the upstream pressure resistance. An airflow honeycomb (position 5) is installed $1637 \mathrm{~mm}$ downstream of the auxiliary fan to uniformly straighten the incoming flow.

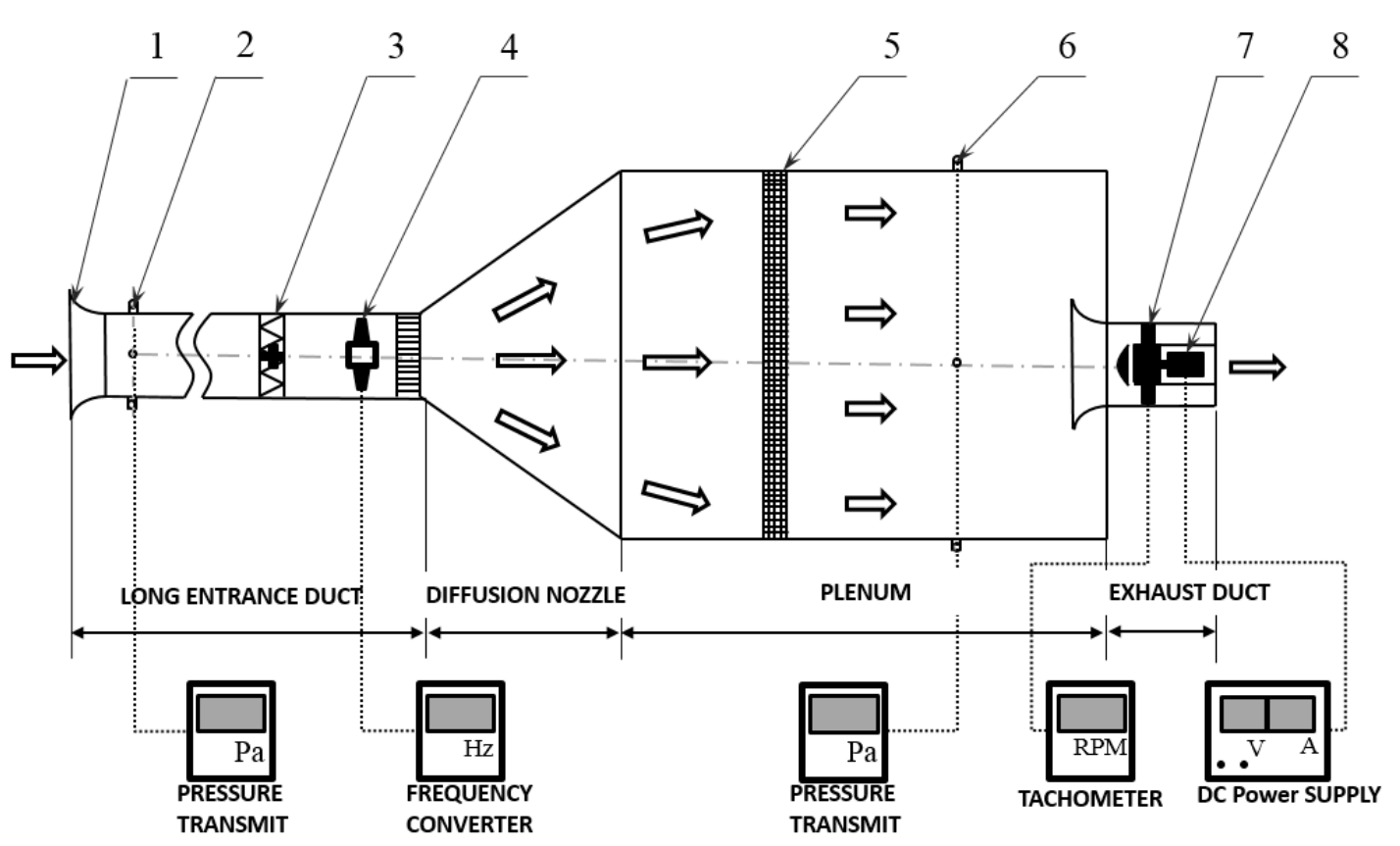

1-Flow Nozzle, 2- Measurement point of flow rate, 3- Flowrate regulating valve 4-Auxiliary Fan, 5- Airflow honeycomb, 6- Measurement point of inlet static pressure to the fan 7- Testing Fan, 8- Motor 


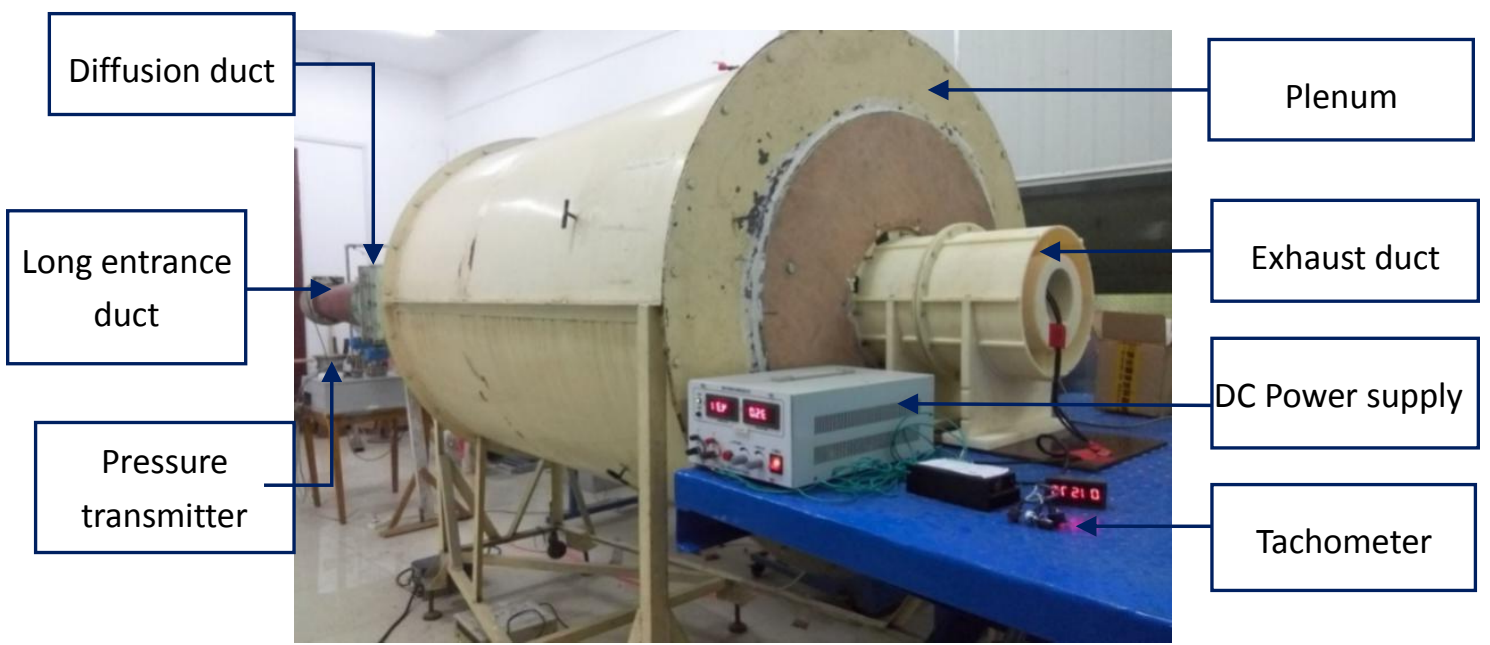

The testing fan is installed inside the exhaust duct, as shown in Figure 2. The exhaust duct consists

123 of four parts (inlet collector, hub fairing, fan and motor cartridge). Using this design, the tested

cartridge and one of four optional fans. The fan is the only component that must be changed for

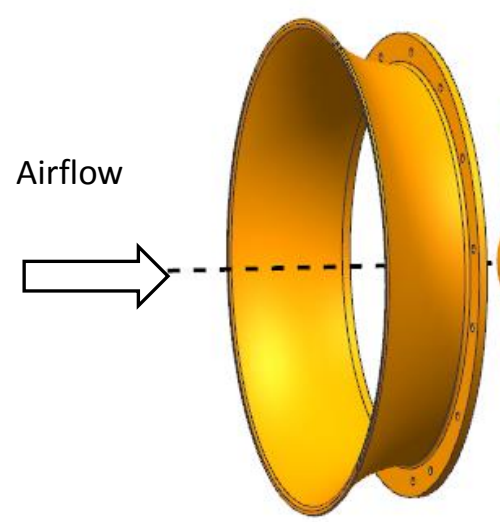

Inlet collector

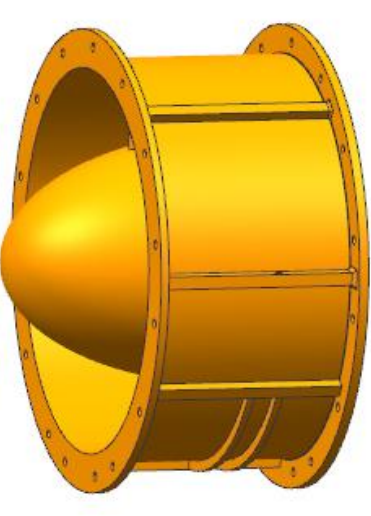

Hub fairing

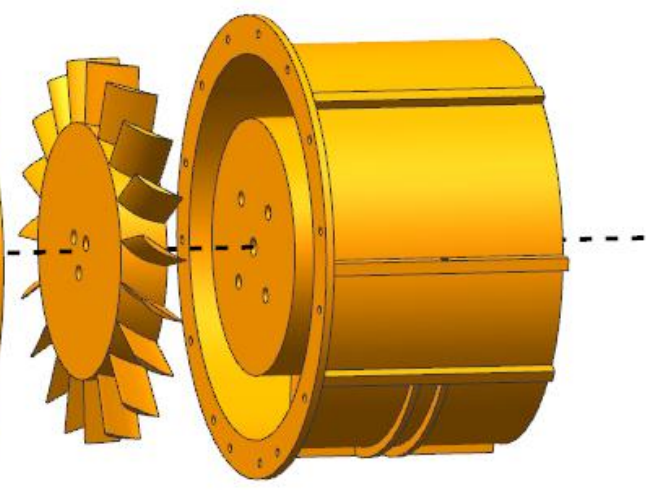

Fan
Motor cartridge 


\subsection{Fan Models}

132

133

134

135

136

137

One prototype fan and three fans with GFs are tested in this paper. They are axial fans with 19 straight blades and have a low aspect ratio. This type of fan is commonly used for room ventilation and air-conditioning systems. Generally, fans with GFs have the same geometrical sizes as the prototype fan. The mean section airfoil of the fan blades is a NACA 65-(12)10 airfoil with a height of $49 \mathrm{~mm}$ and a chord length of $41 \mathrm{~mm}$. The diameter of the shroud and hub is $D_{t}=300 \mathrm{~mm}$ and $D_{h}=200 \mathrm{~mm}$, respectively. The tip clearance is $1 \mathrm{~mm}$, and the designed rotational speed is $1450 \mathrm{RPM}$. More information about these axial fans can be found in Table 1.

Table 1 The design parameters of the fan

\begin{tabular}{lcll}
\hline Rotational speed $(\mathrm{rpm})$ & 1450 & Shroud Diameter $(\mathrm{mm})$ & 300 \\
Rated flowrate $\left(\mathrm{m}^{3} / \mathrm{h}\right)$ & 2191 & Hub ratio & 0.667 \\
Design pressure rise $(\mathrm{Pa})$ & 98 & Hub Diameter $(\mathrm{mm})$ & 200 \\
Blade stagger angle $\left(^{\circ}\right)$ & 45.8 & Incidence $\left(^{\circ}\right)$ & 14.2 \\
\hline Efficiency & $50 \%$ & Blade number & 19 \\
\hline
\end{tabular}

We add the GF directly to the trailing edge of the blade of the prototype fan to form an axial fan with a GF. Three flaps with different heights $1.25 \%, 2.5 \%$ and $5 \%$ of chord length are studied, which means that the height of the GFs are only $0.51 \mathrm{~mm}, 1.03 \mathrm{~mm}$ and $2.05 \mathrm{~mm}$. This is too small of a dimension to be fabricated by a CNC. Thus, during testing, a special type of tape was adopted to form the GFs. Figure 3 presents the real models of axial fans with and without GFs. The special black tape has different thicknesses, and we can choose an appropriate thickness to form the GFs. 


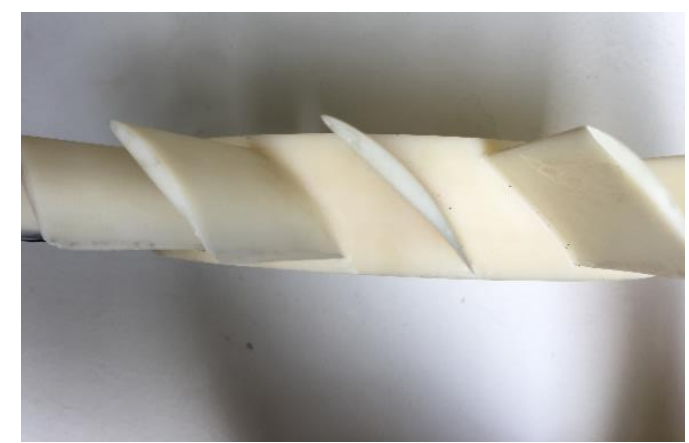

147

\subsection{Data acquisition devices and reduction}

\subsubsection{Data acquisition}

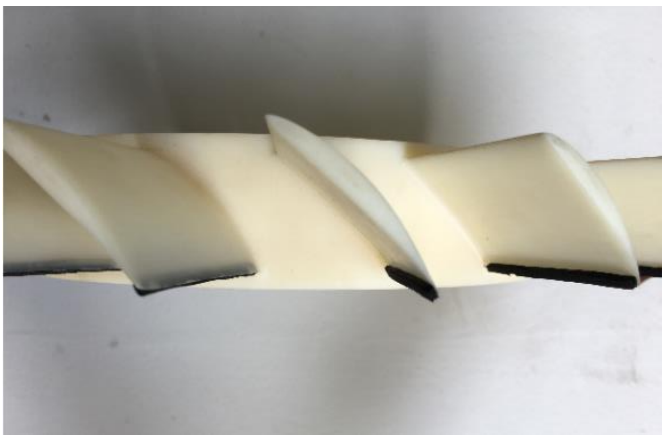

GF Fan

The data acquisition devices used are mainly a pressure transmitter, thermometer, hygrometer tachometer, and torquemeter. First, the pressure, temperature and humidity of the surrounding environment $\left(P_{\mathrm{a}}, T_{\mathrm{a}}\right.$ and $\left.h\right)$ are recorded. Second, one pressure transmitter with an accuracy of $\pm 0.2 \%$ placed at point 2 in Figure 1 is used to measure the static pressure $\left(p_{2}\right)$ at the wind tunnel entrance to calculate the volumetric flowrate $\left(q_{m}\right)$. The total inlet pressure $\left(P_{t, i n}\right)$ of the fan is measured by a second pressure transmitter at point 6 in Figure 1. The static pressure in the plenum can be regarded as the total pressure since the airflow speed is controlled to less than $1 \mathrm{~m} / \mathrm{s}$. The rotational speed of the fan $(N)$ is gained by a tachometer with an accuracy of $0.1 \% \pm 1$ significant digit. The power $\left(P_{e}\right)$ to the motor is obtained through the torquemeter. Table 2 presents detailed information about the data acquisition devices, including the name, range and accuracy. 


\begin{tabular}{|c|c|c|c|}
\hline Name & Measured parameters & Range & Accuracy \\
\hline & Inlet duct static pressure & $0 \sim 1500 \mathrm{~Pa}$ & $\pm 0.2 \%$ \\
\hline & Chamber static pressure & $0 \sim 1500 P a$ & $\pm 0.2 \%$ \\
\hline Torquemeter & Power of Motor & $0 \sim 500 W$ & $\pm 0.5 \%$ \\
\hline Tachometer & Motor Rotational speed & $30 \sim 9999$ rpm & $\begin{array}{l}0.1 \% \pm 1 \text { significant } \\
\text { digit }\end{array}$ \\
\hline \multirow[t]{2}{*}{ Thermometer } & Ambient Temperature & $-40 \sim 60^{\circ} \mathrm{C}$ & $\pm 0.6^{\circ} \mathrm{C}$ \\
\hline & & & $\pm 3 \% R H(0 \sim 90 \% R H)$ \\
\hline Hygrometer & Ambient Humidity & $0 \sim 100 \% R H$ & $\pm 5 \% R H(0 \sim 100 \% R H)$ \\
\hline
\end{tabular}

\subsubsection{Data reduction}

164 The purpose of fan testing is to obtain a performance map of the fan, including the pressure rise,

165 efficiency, and shaft power as a function of the flowrate or rotational speed. Through the above

166 data acquisition system, we can easily obtain values for $P_{\mathrm{a}}, T_{\mathrm{a}}, h, p_{2}, p_{6}, P_{t, i n}, N$ and $P_{e}$.

167 The static pressure through the inlet nozzle is given by

$168 \Delta p=P_{a}-P_{2}$

169 The mass flow rate can be calculated by the following formula using elementary mass conservation

170 and Bernoulli's law

$q_{m}=\alpha \varepsilon \frac{\pi d_{i n}^{2}}{4} \sqrt{2 \rho_{a} \Delta p}$

172 where $\alpha$ is a constant to account for the nozzle discharge coefficient and can be obtained from the 
173 Chinese national standard of fan measurement (GB/T1236-2000); $\varepsilon$ is the expansion coefficient

174 of the nozzle, $\varepsilon=1-0.55 \frac{T_{a}}{P_{a}} ; T_{a}$ is the atmospheric temperature; $P_{a}$ is the atmospheric

175 pressure; $\mathrm{d}_{\mathrm{in}}$ is the flowmeter differential pressure measurement section diameter; and $\rho_{a}$ is

176 the air density in the ambient environment.

177 The total pressure rise of the fan can be expressed in this form:

$178 \Delta P=P_{t, \text { out }}-P_{t, \text { in }}=P_{t, \text { out }}-P_{6}$

179 where $P_{t, \text { out }}$ is defined by

$180 P_{t, \text { out }}=P_{a}+P_{d, \text { out }}$

181 and $P_{d, \text { out }}$ is the dynamic pressure of the exhaust airflow of the fan. Considering the balance of

182 mass flowrate, $P_{d, \text { out }}$ is given by

$P_{d, \text { out }}=\frac{1}{2} \rho_{2}\left(\frac{q_{m}}{\rho_{2} A_{2}}\right)^{2}$

where $q_{m}$ is the mass flowrate of the air, $A_{2}$ is the outlet area, $\rho_{2}$ is the density of the outlet air, and $\rho_{2}=\rho_{a}$ due to the incompressibility assumption for the low velocity.

186 The static pressure rise of the fan can be expressed in the form:

187

$\Delta p=P_{a}-P_{i n}=P_{a}-P_{6}$

The total pressure rise coefficient (TPRC) $C_{p . t}$, static pressure rise coefficient (SPRC) $C_{p . s}$, flow

191

$$
C_{P, t}=\frac{\Delta P}{\rho u^{2}}
$$


192

$C_{P, S}=\frac{\Delta p}{\rho u^{2}}$

193

$\bar{q}=\frac{q_{m}}{\rho u A}$

$194 \quad \eta_{\mathrm{t}}=\frac{\Delta P \times \mathrm{q}_{V}}{P_{e} \times \eta_{\mathrm{e}}}$

$195 \quad \eta_{\mathrm{s}}=\frac{\Delta p \times q_{V}}{P_{e} \times \eta_{e}}$

196 where $q_{v}$ is the volume flowrate and $\eta_{e}$ is the efficiency of the motor.

\subsection{Uncertainty analysis}

According to error theory, measurement error is composed of system error, random error and gross

error. The experimental data in this paper is mainly obtained by the data acquisition devices. The

instrument error.

Assume that the directly measured quantities are $\mathrm{x}_{1}, \mathrm{x}_{2} \ldots, \mathrm{X}_{\mathrm{m}}$ and the indirectly measured quantity is $y$. The functional relation between them is:

$y=f\left(x_{1}, x_{2}, x_{3}, \ldots, x_{m}\right)$

If the direct measurement errors are $\Delta x_{1}, \Delta x_{2}, \ldots, \Delta x_{m}$, they have affected the indirectly

$$
\Delta \mathrm{y}=\frac{\partial f}{\partial x_{1}} \Delta x_{1}+\frac{\partial f}{\partial x_{2}} \Delta x_{2}+\ldots+\frac{\partial f}{\partial x_{m}} \Delta x_{m}=\sum_{i=1}^{m} \frac{\partial f}{\partial x_{i}} \Delta x_{i}
$$


$211 \quad L^{2}=\sum_{i=1}^{m}\left(\frac{\partial f}{\partial x_{i}} \Delta x_{i}\right)^{2}$

$$
R=\frac{287}{1-\frac{0.378 \times h \times P_{u}}{P_{a}}}
$$

$214 P_{a}$ is the atmospheric pressure and $h$ is the relative humidity. $P_{u}$ is the saturation steam

215 pressure.

$$
P_{u}=\exp \left(\frac{17.438 T_{a}}{239.78+T_{a}}+6.4147\right)
$$

$217 T_{a}$ is the atmospheric temperature.

218 The error of the gas constant can be calculated by the following formula:

$$
L_{R}=\sqrt{\left(\frac{\partial R}{\partial h} \times \Delta h\right)^{2}+\left(\frac{\partial R}{\partial P_{a}} \times \Delta P_{a}\right)^{2}+\left(\frac{\partial R}{\partial P_{u}} \times \Delta P_{u}\right)^{2}}
$$

\section{Atmospheric density}

$$
\rho_{a}=\frac{P_{a}}{R\left(273.15+T_{a}\right)}
$$

222 The error of the atmospheric density can be calculated by the following formula:

$$
L_{\rho}=\sqrt{\left(\frac{\partial \rho_{a}}{\partial P_{a}} \times \Delta P_{a}\right)^{2}+\left(\frac{\partial \rho_{a}}{\partial R} \times \Delta R\right)^{2}+\left(\frac{\partial \rho_{a}}{\partial t_{a}} \times \Delta T_{a}\right)^{2}}
$$

$$
q_{V}=\frac{q_{m}}{\rho_{a}}=\frac{\alpha \varepsilon \frac{\pi d_{\text {in }}^{2}}{4} \times \sqrt{2 \Delta p}}{\sqrt{\rho_{a}}}
$$




$$
L_{q}=\sqrt{\left(\frac{\partial q_{V}}{\partial \Delta P} \times \Delta P\right)^{2}+\left(\frac{\partial q_{V}}{\partial \rho_{a}} \times \Delta \rho_{a}\right)^{2}}
$$

228 4. Exhaust airflow density

229

$$
\rho_{2}=\frac{P_{a}}{R\left(273.15+t_{2}\right)}
$$

$$
t_{2}=T_{a}+\frac{P_{e} \times \eta_{\mathrm{e}}}{q_{V} \times \rho_{a} \times c_{p}}
$$

$231 c_{p}$ is the specific heat.

232 The error of the exhaust airflow density can be calculated by the following formula:

$$
\mathrm{L}_{\rho_{2}}=\sqrt{\left(\frac{\partial \rho_{2}}{\partial P_{a}} \times \Delta P_{a}\right)^{2}+\left(\frac{\partial \rho_{2}}{\partial R} \times \Delta R\right)^{2}+\left(\frac{\partial \rho_{2}}{\partial t_{2}} \times \Delta t_{2}\right)^{2}}
$$

$$
\Delta P=P_{t, \text { out }}-P_{t, \text { in }}
$$$$
P_{t, \text { out }}=P_{a}+P_{d, \text { out }}
$$$$
P_{d, \text { out }}=\frac{1}{2} \rho_{2}\left(\frac{q_{V}}{A_{2}}\right)^{2}
$$

$236 P_{t, \text { out }}$ is the exit total pressure. $P_{t, \text { in }}$ is the inlet total pressure, and $P_{d, \text { out }}$ is the exit dynamic pressure.

238 The error of the exhaust airflow density can be calculated by the following formula:

$$
L_{\Delta P}=\sqrt{\left(\frac{\partial \Delta P}{\partial P_{t, \text { out }}} \times \Delta P_{t, \text { out }}\right)^{2}+\left(\frac{\partial \Delta P}{\partial P_{t, \text { in }}} \times \Delta P_{t, \text { in }}\right)^{2}}
$$

$241 \quad \eta_{\mathrm{t}}=\frac{q_{V} \times \Delta P}{P_{e} \times \eta_{\mathrm{e}}}$ 
$L_{\eta r}=\sqrt{\left(\frac{\partial \eta_{r}}{\partial P_{e}} \times \Delta P_{e}\right)^{2}+\left(\frac{\partial \eta_{r}}{\partial q_{V}} \times \Delta q_{V}\right)^{2}+\left(\frac{\partial \eta_{r}}{\partial P} \times \Delta P\right)^{2}}$

244 The final results are shown in Table 3.

Table 3 Error range of the calculation parameters

\begin{tabular}{|l|l|l|}
\hline & Measurement parameters & Comprehensive accuracy \\
\hline 1 & Gas constant & \pm 0.796 \\
\hline 2 & Atmospheric density & $\pm 0.3 \%$ \\
\hline 3 & Volume flow & $\pm 0.42 \%$ \\
\hline 4 & Fan outlet air density & $\pm 0.03 \%$ \\
\hline 5 & Fan total pressure & $\pm 4.69 \mathrm{~Pa}$ \\
\hline & Fan efficiency & $\pm 1.6 \%$ \\
\hline
\end{tabular}

\section{Numerical method}

\section{$247 \quad 3.1$ Computational domain modeling}

248 It is recommended that the computational domain be the image of the actual tested conditions.

Hence, we created the computational domain including the upstream domain (plenum), axial fan

250 and downstream domain (ambient environment). The upstream and downstream domains are extended six times the diameter of the fan for better steadiness of flow. The whole computational domain can be found in Figure 4. The GF size, especially the height, is relatively smaller than the size of the other parts. It is difficult to resolve this small feature in a large computational domain 
255

256

257

tetrahedral mesh. Boundary layers are paved on the solid wall surfaces of the blades, hub and shroud to ensure that $y$-plus is less than 1 to resolve the near-wall flow properly. The mesh size is also refined in the areas that are close to the axial fan to properly adapt to the large gradient variation of the pressure and velocity. The detailed mesh around the fan blade is shown in Figure 4.
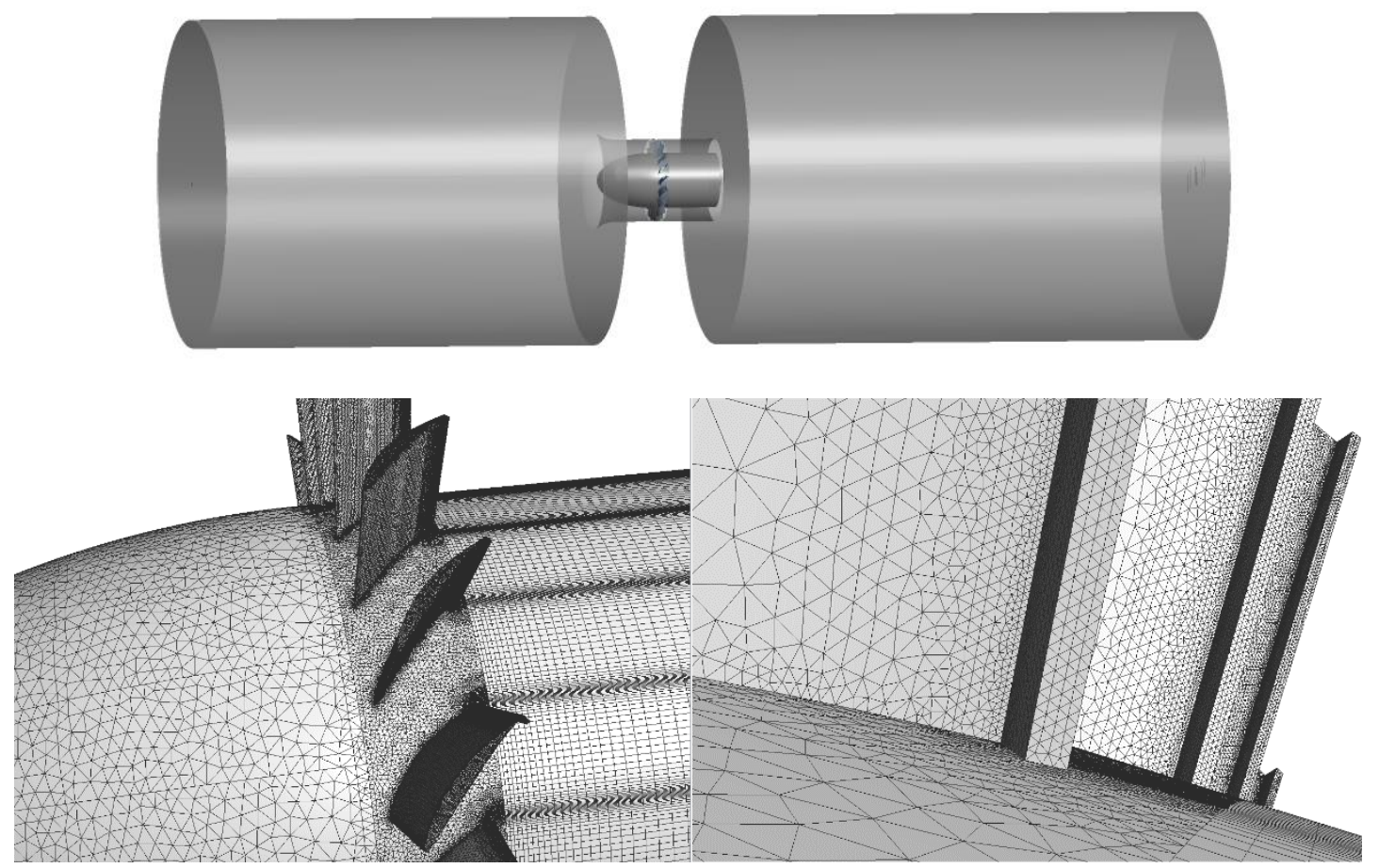

Figure 4 Detailed mesh around the fan blade.

\subsection{Governing equations and simulation strategies}

The flow through fan is taken as turbulent incompressible flow and is governed by the Reynolds-averaged Navier-Stokes (RANS) equations,

$\frac{\partial \bar{u}_{i}}{\partial x_{i}}=0$

where $\bar{u}_{i}$ is the averaged velocity component in the ith-direction. 
268

$\frac{\partial\left(\rho \bar{u}_{i}\right)}{\partial t}+\frac{\partial}{\partial x_{j}} \rho \overline{u_{i}^{\prime} u_{j}^{\prime}}=-\frac{\partial \bar{p}}{\partial x_{i}}+\mu \frac{\partial^{2} \bar{u}_{i}}{\partial x_{j} \partial x_{j}}+\rho \bar{F}_{i}$

$-\rho \overline{u_{i}^{\prime} u_{j}^{\prime}}=-\mu_{t}\left(\frac{\partial \bar{u}_{i}}{\partial x_{j}}+\frac{\partial \bar{u}_{j}}{\partial x_{i}}\right)-\frac{2}{3} \delta_{i j}\left(\rho k+\mu_{t} \frac{\partial \bar{u}_{i}}{\partial x_{j}}\right)$

273 where $\mu_{t}$ denotes eddy viscosity and $\mathrm{k}$ is the turbulent kinetic energy and is defined as [5]:

$k=\frac{1}{2} \rho \overline{u_{i}^{\prime} u_{j}^{\prime}}(4)$

The above equations need turbulence closure for resolution. The RNG k- $\varepsilon$ turbulence model and

SIMPLE algorithm were applied to the steady simulation calculation of the flow field in the small axial flow fan [23-25]. The shear-stress-transport (SST) turbulent model was adopted by Kim to calculate the flow field of a tunnel ventilation jet fan[26]. This turbulence model is sensitive to adverse pressure gradients and boundary layer separation, which are more common when an axial fan is under a state of rotational periodicity. Generally, in the case of shear-stress-transport (SST) turbulent models or $k-\omega$ turbulent models, using the wall function is known to be valid when the y-plus values are not higher than 300 [7]. The shear-stress-transport (SST) turbulent model, which has the advantages of both $k-\omega$ and k- $\varepsilon$, employs the $k-\omega$ model at the near-wall and the k- $\varepsilon$ model in the bulk-flow region, a blending function that ensures a smooth transition between the two models [27]. Since the SST model showed better predictions of flow separation along smooth surfaces [27], it was adopted for use in this paper. 
288 interpolation schemes are applied for the pressure and momentum equation, respectively. The

289 SIMPLE algorithm is adopted to handle the pressure-velocity coupling. The frozen rotor method is

290 utilized to address the data transform between the rotational and stationary zone interface. A

291 convergence criterion is prescribed to obtain a periodic variation of the torque of the fan as well

292 as a maximum residual less than $1.0 \times 10^{-5}$.

\section{Results and Discussion}

\subsection{Performance of the Fan}

295 Figure 5 shows the effect of the height of the GF on the total pressure rise coefficient $\left(C_{P, \mathrm{t}}\right)$ of the 296 fan. It is clear that $C_{P, \mathrm{t}}$ increases as the GF height increases. Compared with the original fan at $\bar{q}=0.30, C_{P, \mathrm{t}}$ increases by $6 \%, 12.23 \%, 13.54 \%$ for three GFs heights. As the flow coefficient increases, the magnitude of the total pressure rise coefficient increase is becoming larger. It indicates that a higher GF produces a higher total pressure and maximum operating flow range. In other words, a fan with a GF has a large operating flow range. The higher total pressure is also 


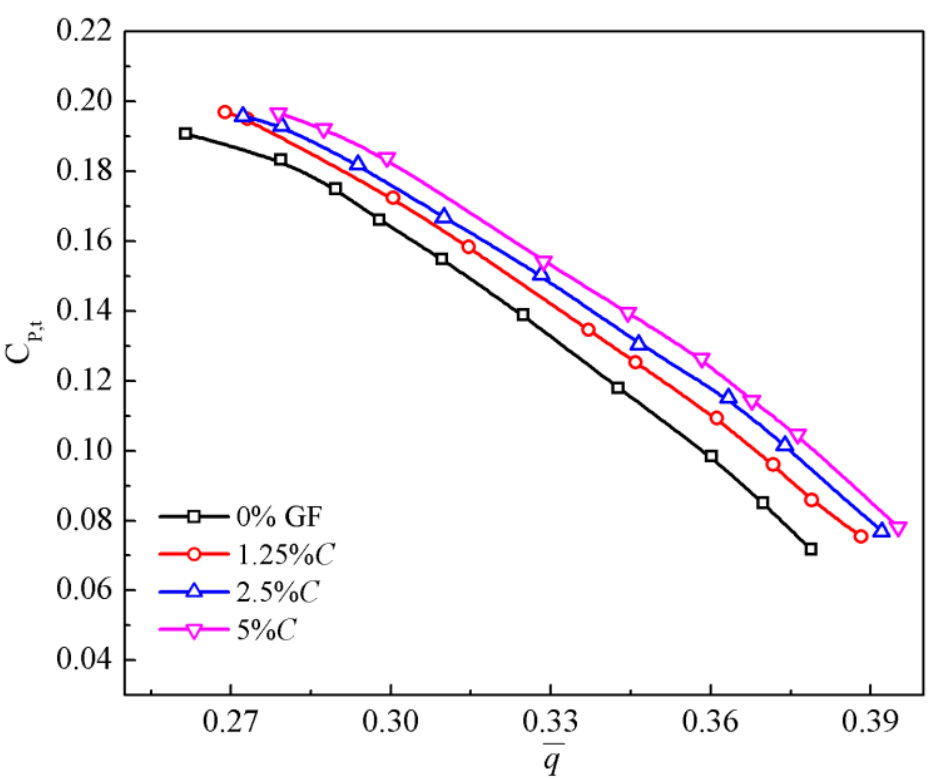

302

304 Figure 6 shows the effects of the height of the GF on the total pressure efficiency $\left(\eta_{t}\right)$ at different

305 flow coefficients. It is found that the total pressure efficiencies of fans with GFs are always lower 306

Figure 5 Effects of GF height on the total pressure rise coefficient

than that of the original. The difference between the total pressure efficiency curves is not obvious in the flow coefficient range from 0.14 to 0.27 for fans with $1.25 \%$ and $2.5 \%$ chord GFs. As the flow coefficient increases to 0.39 , the difference among the four curves is increased. The total pressure efficiencies at $\bar{q}=0.30$ for $1.25 \%, 2.5 \%, 5 \%$ chord GFs are $1.76 \%, 4.6 \%$, and $6.7 \%$ lower than that of the original fan, respectively. All of the above observations indicate that GFs with $1.25 \%$ and $2.5 \%$ chords have very small effects on the total pressure efficiency at low flow coefficients. As the flow coefficient reaches a certain value, the influence of the height of the GF on the total pressure efficiency is remarkable. 


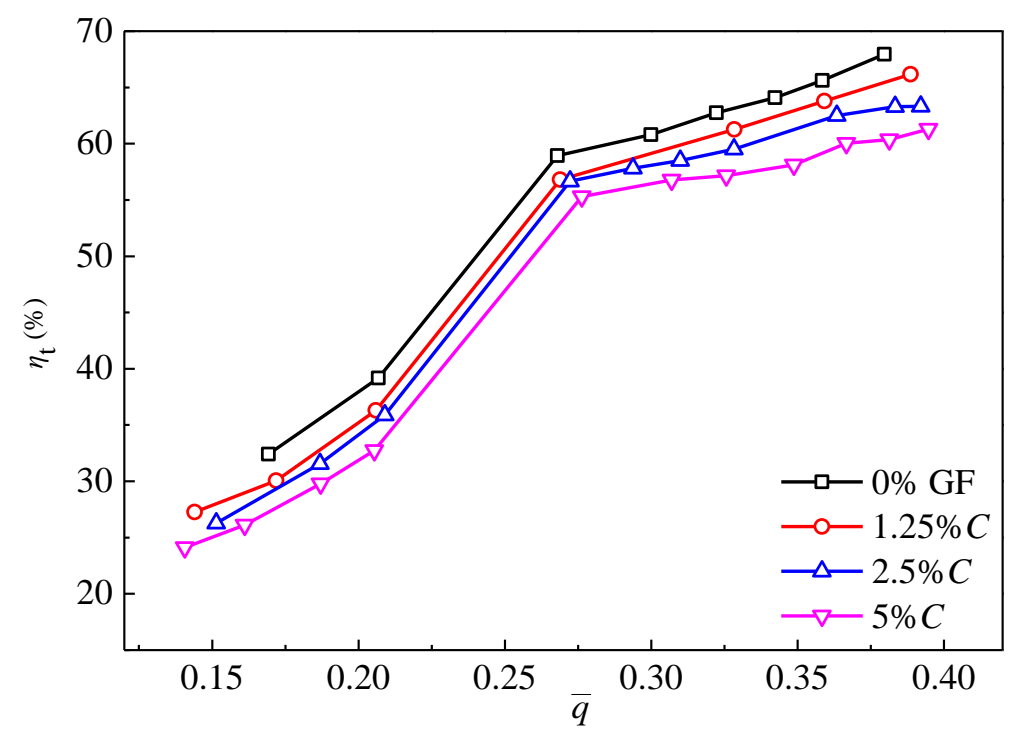

314

Figure 6 Effects of the GF height on the total pressure efficiency

The effect of the GF height on the static pressure efficiency $\left(\eta_{S}\right)$ is presented in Figure 7. It decreases with the increasing height of the GF in the flow coefficient range from 0.12 to 0.27 . The difference among the four curves of the static pressure efficiency is clear when the flow coefficient varies from 0.12 to 0.27 . This result is different from the conclusion determined from total pressure efficiency. The maximum static pressure efficiencies of fans with $1.25 \%, 2.5 \%, 5 \%$ chord GFs are $1.04 \%, 1.65 \%$, and $3.05 \%$ lower than that of the original fan, respectively. One possible reason for the efficiency reduction may be the vortex formed by the GF at the trailing edge of the airfoil, producing the loss. Another reason may be that the increased pressure difference between two blade surfaces leads to an increase in clearance leakage. However, when the flow coefficient becomes greater than 0.27 , the opposite behavior is observed, in that the efficiency difference becomes negligible with an increase in the height of the GF. Similar observations are found in the studies of Manish et al [28].

In some application circumstances of cooling, ventilation, vacuuming, dust removal and inflating, 
329

330

331 improvement of the work capability is through this simple modification of adding a GF.

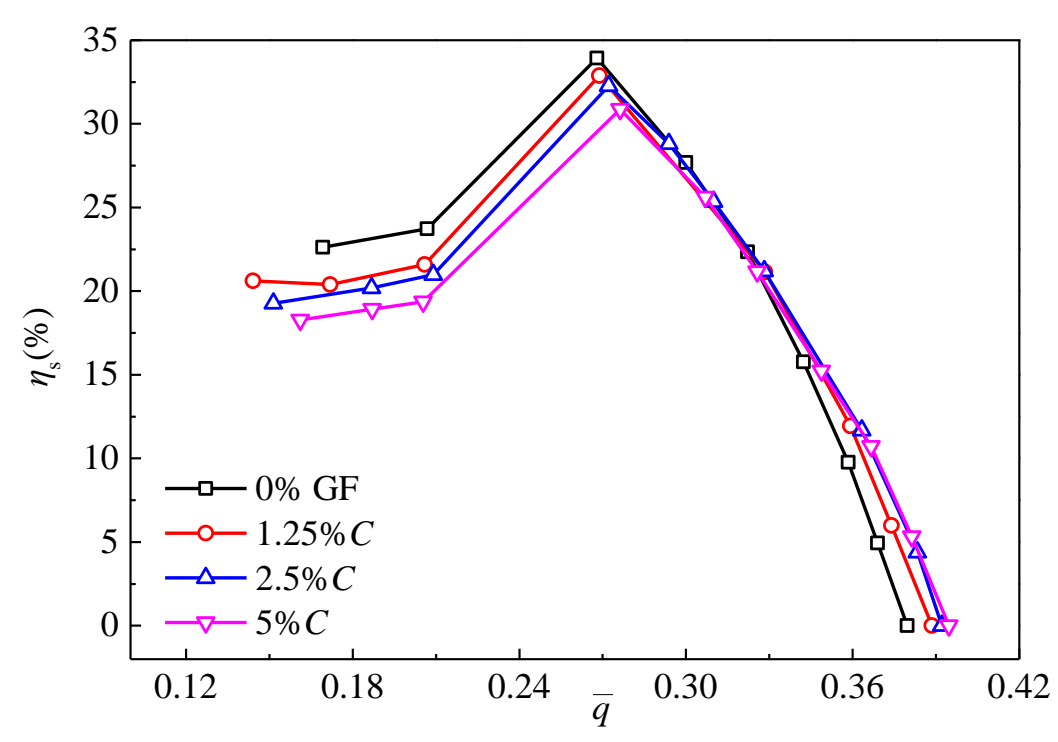

332

333

we need to increase the pressure ratio of the fan without a large efficiency loss due to a limitation on space. The size of the fan is highly limited in a confined space. One potential solution for the

Figure 7 Effects of the GF height on the static pressure efficiency

\subsection{Validation of the simulation results}

The simulation results were validated through a comparison of the tested pressure rise coefficients with the computed values. Total pressure rise coefficients from simulations of the original fan and fan with a $1.25 \%$ chord GF were compared with the corresponding results from the same fan. This comparison is presented in Figure 8. The lines present the simulation results. The symbols are the experimental results. It is evident that the computed values of the total pressure rise coefficient agree well with the experimental results over the majority of the range. This proves that the chosen simulation strategies are reliable. It also provides a solid foundation for the flow field analysis in the following paragraphs. 
343

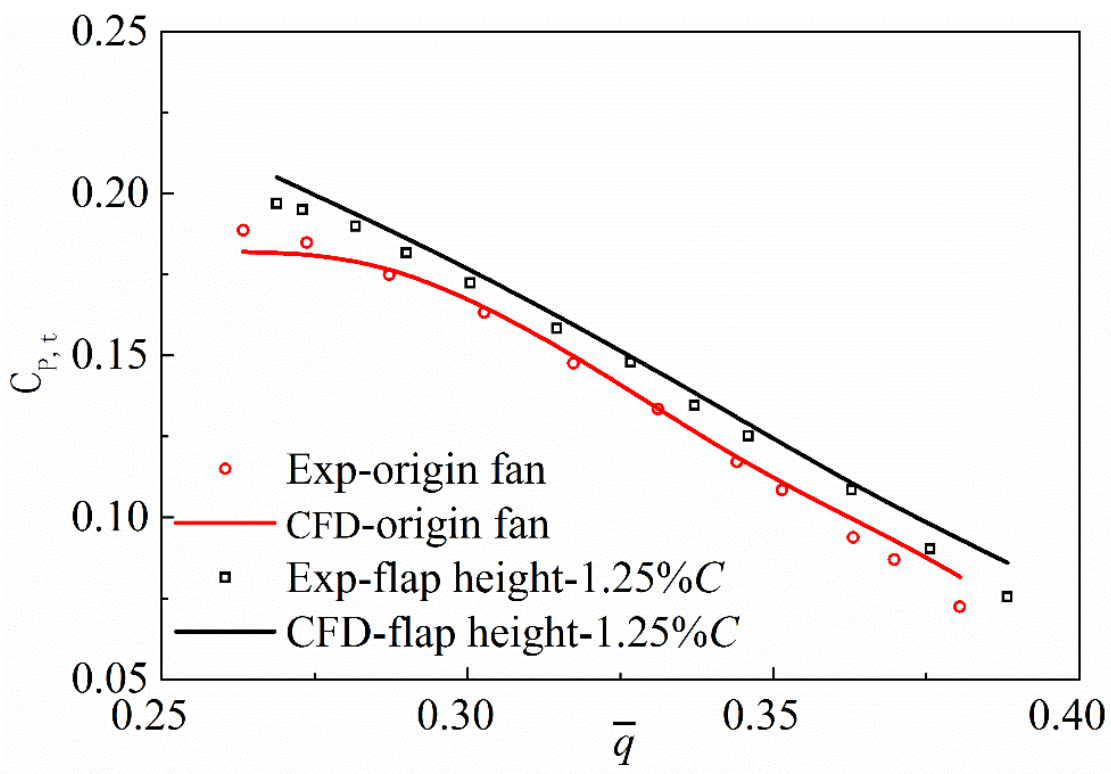

Figure 8 Comparison of the experimental and simulation results

\subsection{Flow analysis of a fan with a GF}

The following numerical simulation was carried out under the condition that the mass flow $\bar{q}$ is

$0.028 \mathrm{~kg} / \mathrm{s}$. Figure 9 shows the distribution of vorticity, which is generated by the Q-criterion at the trailing edge of the airfoil, colored by the static pressure. It is found that the airflow over the original fan is separated from the trailing edge on the suction surface and that a shedding vortex is generated downstream of the trailing edge. When GFs with different heights are added at the trailing edge of the airfoil, it is observed that a low-pressure vortex area is formed to reduce the adverse pressure gradient in the flow channel and accelerate the flow of air. This is a possible reason for the enhancement of the adsorption force at the trailing edge of the suction surface.

\footnotetext{
Thus, the blade load is improved significantly as well as the outlet total pressure.
} 


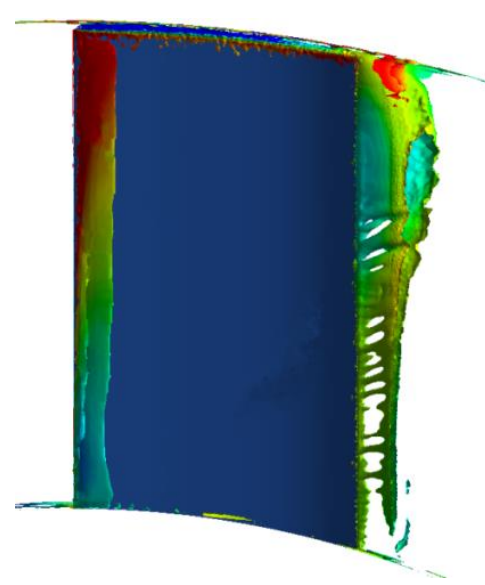

(a) Fan without GF

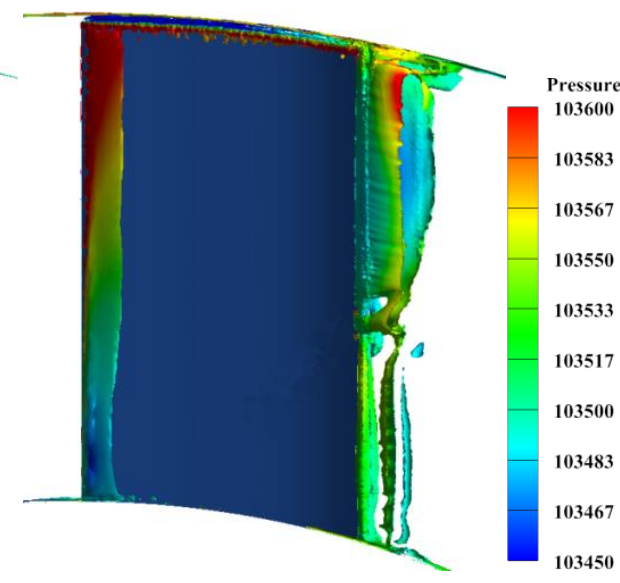

(b) Fan with GF $(1.25 \%$ C)

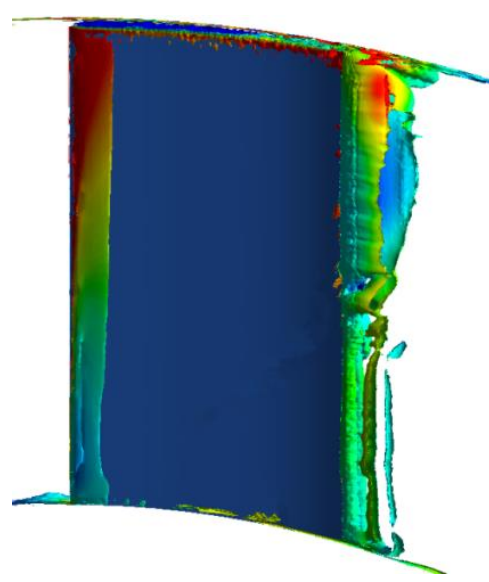

(c) Fan with GF $(2.5 \%$ C

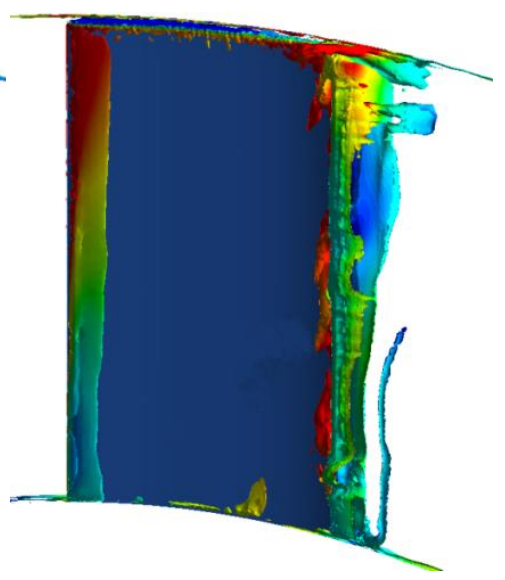

(d) Fan with GF $(5 \%$ C)

Figure 9 Vorticity distribution at the trailing edge of the blade

The distribution of the static pressure on the suction surface is shown in Figure 10. The static pressure on the suction surface is reduced near the trailing edge of the blade as the GF height is increased, which produces the increase in the blade load. At the same time, the distribution of static pressure tends to be smoother. Jeffrey et al. conducted laser Doppler anemometry (LDA) measurements downstream of a Gurney flap. The LDA data shows that the wake consists of a Karman vortex sheet of alternately shedding vortices. When a GF is added at the trailing edge of the airfoil, a periodic shedding of vortices occurs, which reduces the adverse pressure gradient to 
367 368

a certain extent and accelerates the flow over the trailing edge. This results in a decrease in the flow separation and pressure of the suction surface trailing edge. This reduction of flow separation is depicted more intuitively in Figure 12

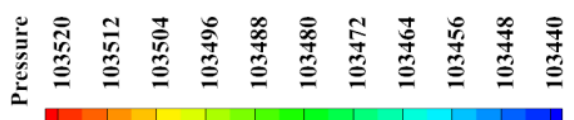

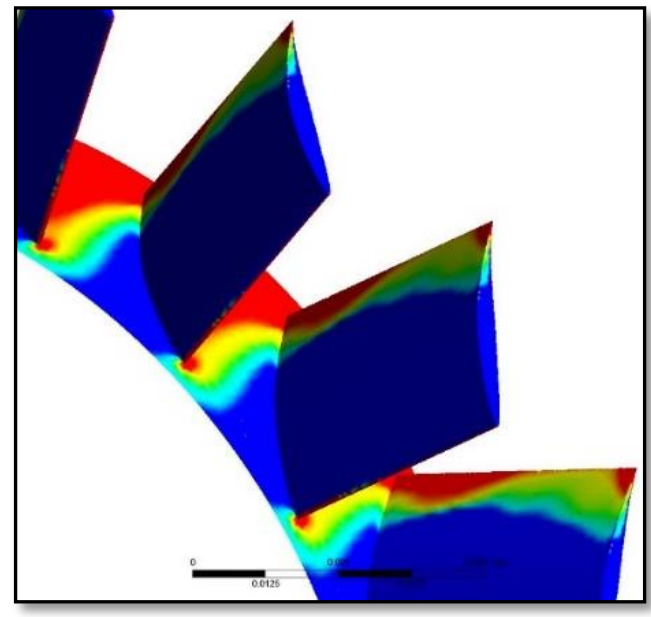

(a) Fan without GF

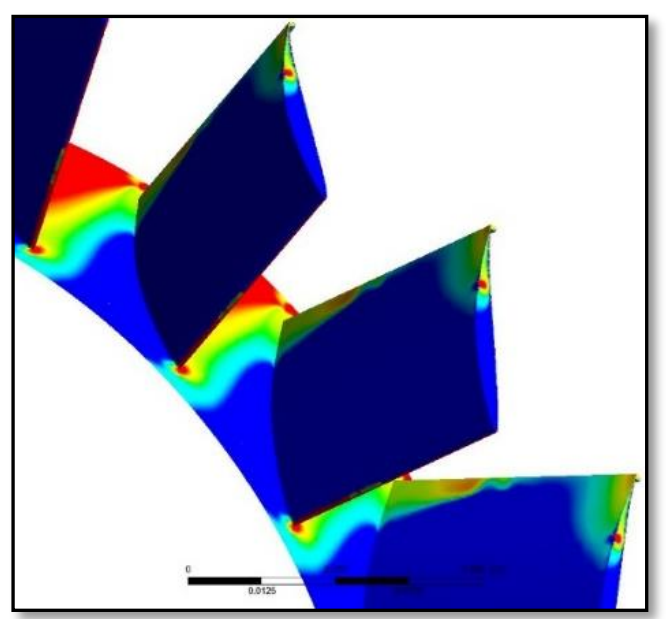

(c) Fan with GF $(2.5 \%$ C)

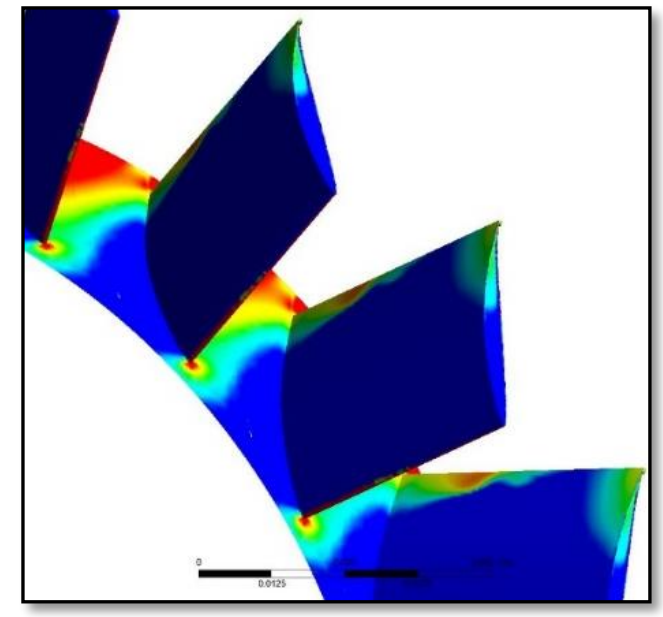

(b) Fan with GF $(1.25 \%$ C)

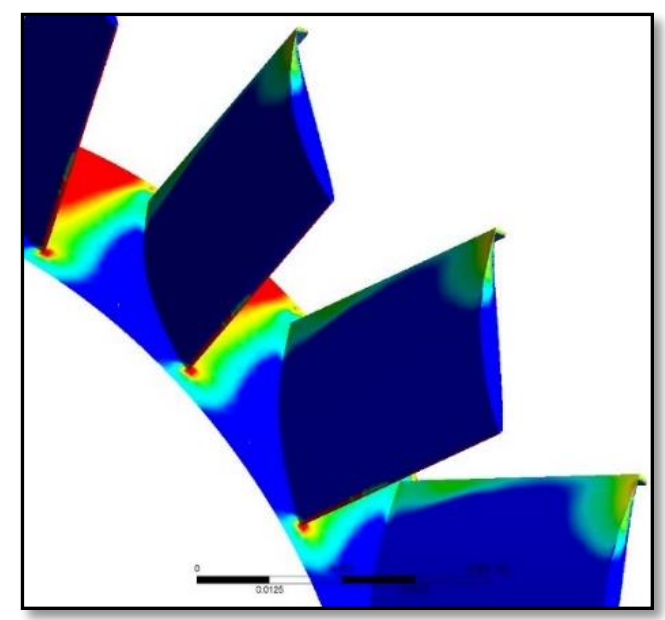

(d) Fan with GF (5\%C)

Figure 10 Static pressure distribution on the suction surface

Figure 11 shows the variation in the total pressure coefficient along the flow direction at the blade midspan. A higher total pressure coefficient is obtained with a higher flap. $33.33 \%$ more total 
378

379

381

382 pressure coefficient can be obtained with the GF height of $5 \% \mathrm{C}$ than the original fan. Since a GF is so limited in size, an obvious difference in the total pressure coefficient between fans with and without GFs can only be found on the near airfoil and trailing edge of the blade.

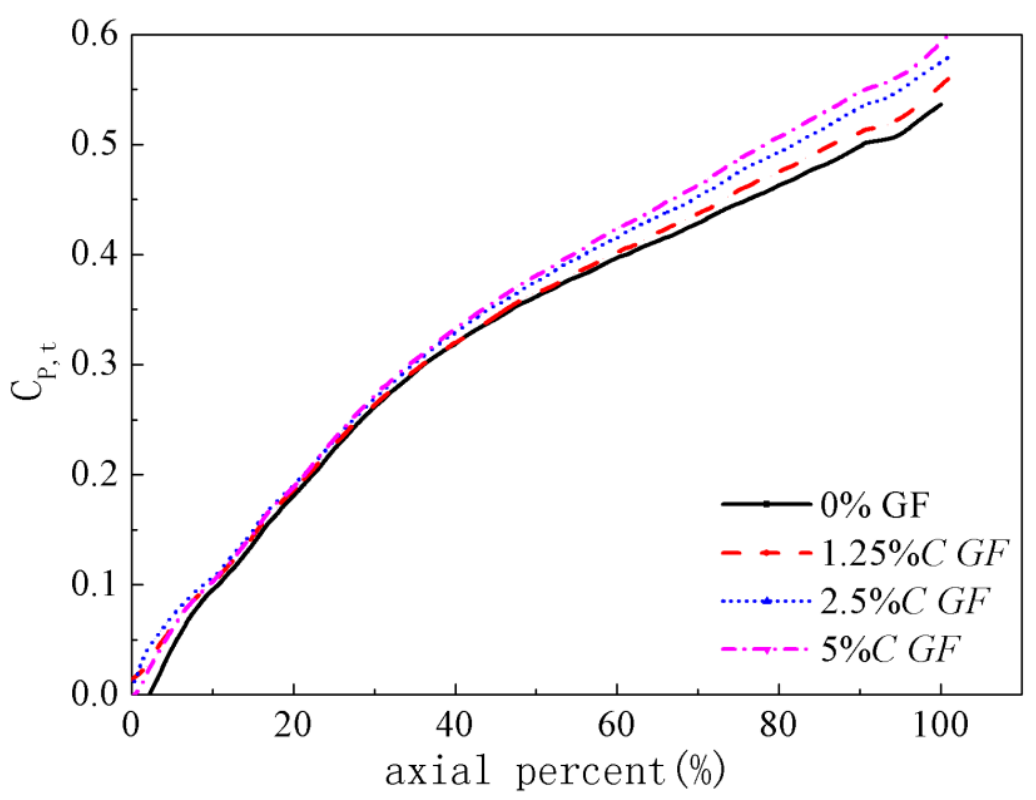

Figure 11 Midspan axial variation of the total pressure rise.

separation occurs earlier on the original fan than on a fan with a GF. This indicates that the flow separation at the suction surface will be suppressed when the GF is installed.

velocity

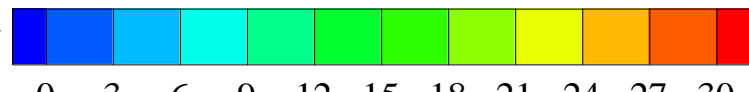

(a) original fan

(b) GF of $1.25 \% C$ height 


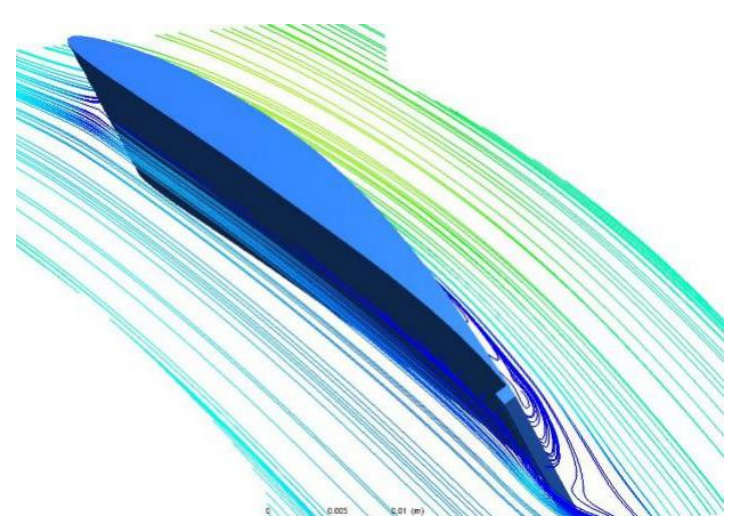

(c) GF of $2.5 \% \mathrm{C}$ height

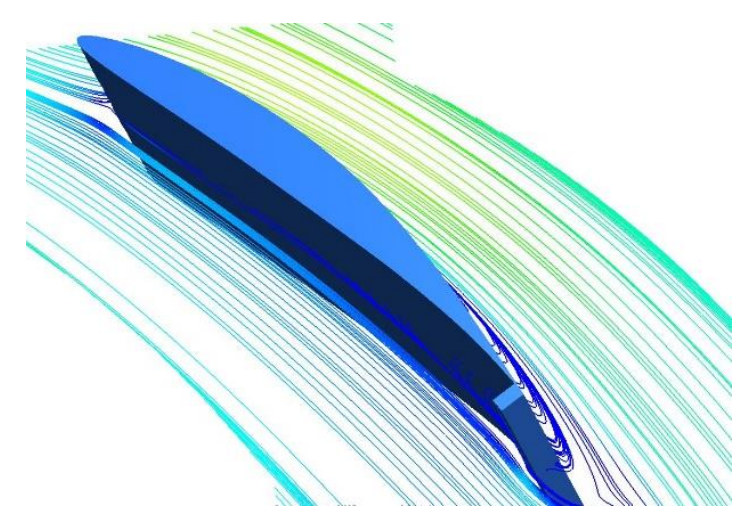

(d) GF of $5 \%$ C height

Figure 12 Streamline distribution on the S1 stream flow surface

Distribution of static pressure at 10\%, 50\%, and $90 \%$ height of the blade is shown in Figure 13

respectively. The dimensionless static pressure is defined as:

$\bar{p}=\frac{P_{1}-P_{i n}}{P_{t, i n}-P_{i n}}$

where $P_{1}$ is the local static pressure on the blade surface. With a GF, the static pressure on the blade suction surface is decreased and the static pressure of the pressure surface is increased, which is visualized clearly close to the blade trailing edge. As presented in Figure 9, shedding vortices with high turbulence intensities are clearly visible in the GF wakes. In addition to those vortices in the wake, an additional vortex region is created in front of the flap. These vortices are responsible for the increase in suction on the upper surface and increased pressure on the lower surface, which enhances the blade lift capability. As the height of the Gurney flap increases, these vortices are strengthened. A larger deflection of the exit flow at the trailing edge is pushed towards the GF, which also causes an increase in the effective downwash and, then, the work capability of the fan. 


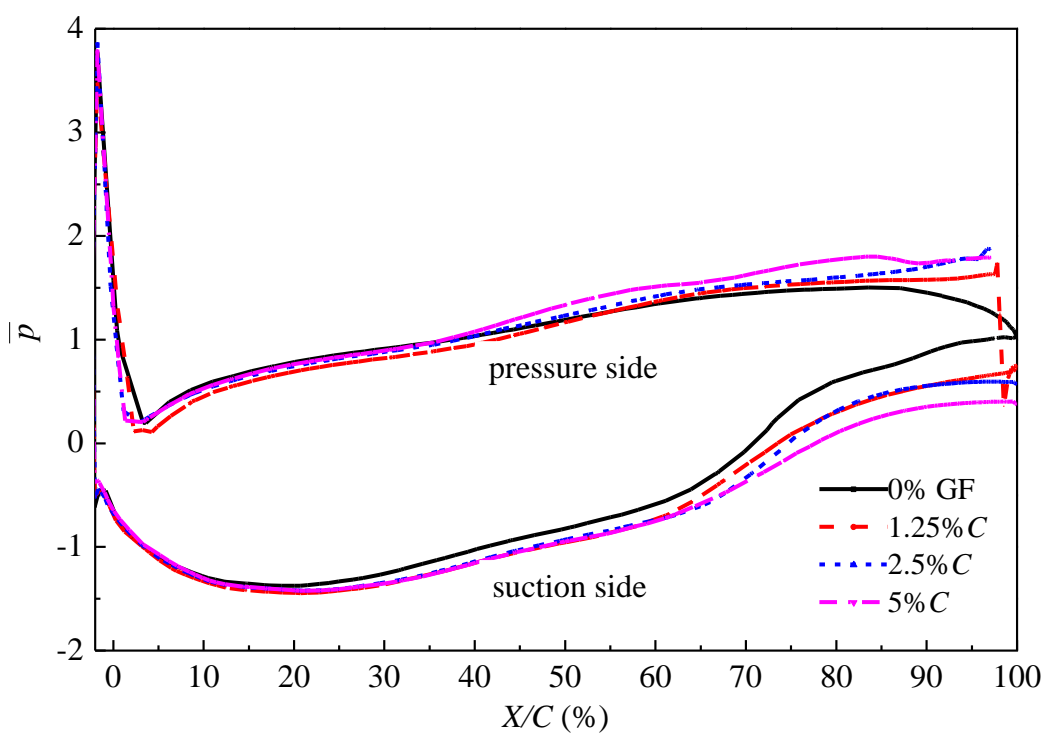

(a) $10 \%$ height of the blade

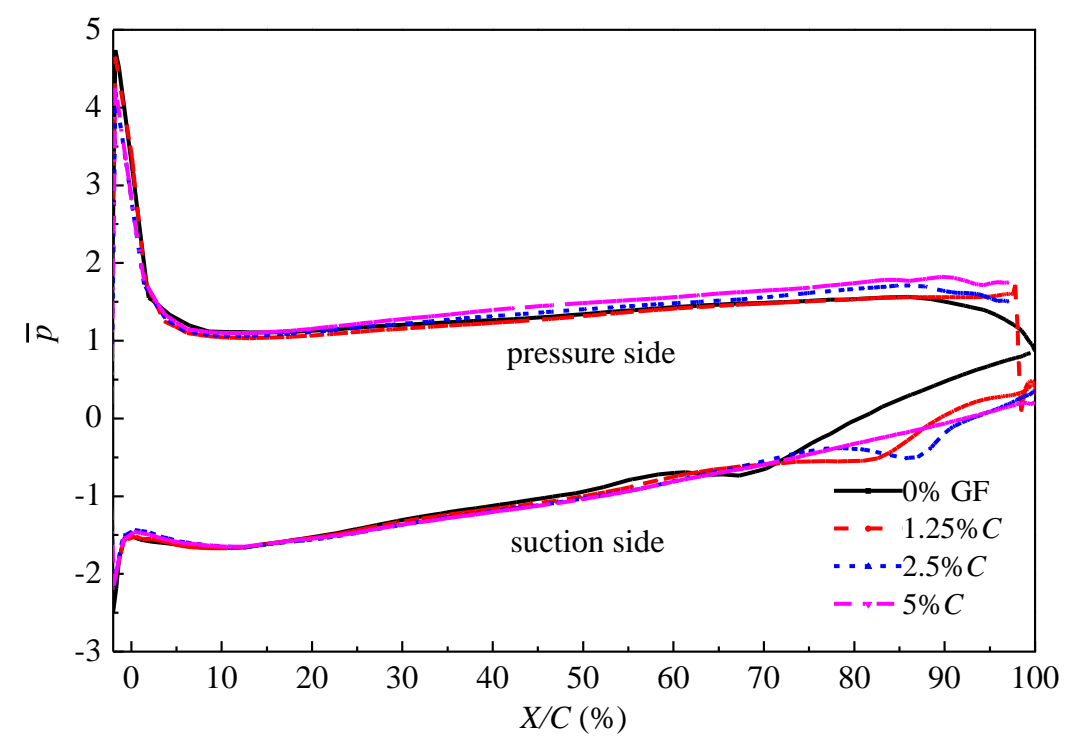

(b) $50 \%$ height of the blade 


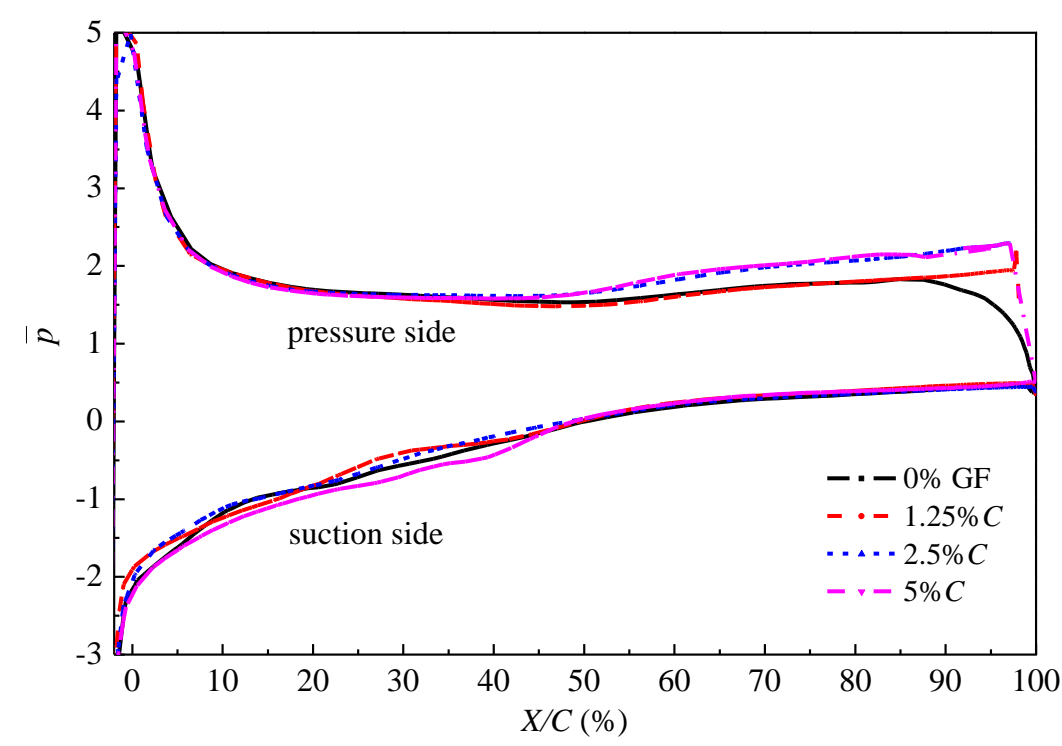

(c) $90 \%$ height of the blade

Figure 13 Static pressure distribution along the blade chord

399 Figure 14 shows the distribution of the pitch-averaged relative outlet airflow angle along the blade

400 height. The relative outlet airflow angle of the fan with a GF is smaller than that of the original fan

401 from the hub endwall to $80 \%$ of the span of the blade, indicating that the flap exerts extra force to

402 push the outlet flow towards the axial direction and cause the flow to have a larger airflow-turning

403 angle. Therefore, the GFs can enhance the work capability of fans. We know from the previous

404 sections that the GF is able to increase the pressure difference between the suction surface and

405 pressure surface, which produces a stronger blade tip leakage vortex, tending to move from the

406 pressure surface to the suction surface. Its mixing with the main flow is intensified with the height

407 of the installed GF. As shown in Figure 14, this difference is clear at the spanwise position from $80 \%$

408 to $100 \%$ of the blade height, where the tip leakage mixes with the main flow region. 


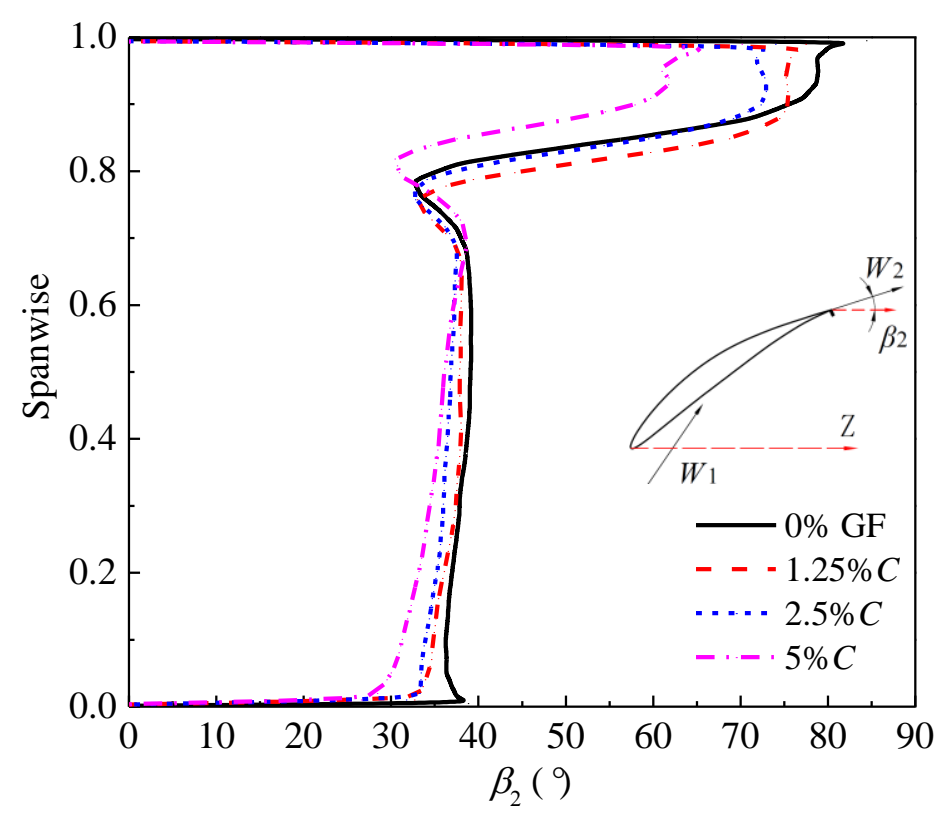

Figure 14 Relative outlet airflow angle along the span

409 Figure 15 shows the variation in the total radial pressure rise at the positions of $0 \%, 20 \%, 40 \%, 60 \%$,

$41080 \%$, and $100 \%$ of the axial chord. Compared with the original fan, there is no obvious difference in

411 the pressure distribution before $20 \%$ of the axial chord. When the flow comes to a position of approximately $40 \%$ of the axial chord, the difference in the total pressure at the blade tip becomes

413 obvious due to the strengthened tip leakage caused by the GF. This phenomenon can be clearly

414 found when the flow runs over the blade surface near $60 \%$ of the chord. At positions of $60 \%, 80 \%$,

415 and $100 \%$ of the axial chord, the total pressure of a fan with a GF is always higher than that of the original fan for the entire blade span. Approaching the blade trailing edge, larger differences in

417 pressure appear. In addition, this difference will be more obvious when the height of the GF is increased. 


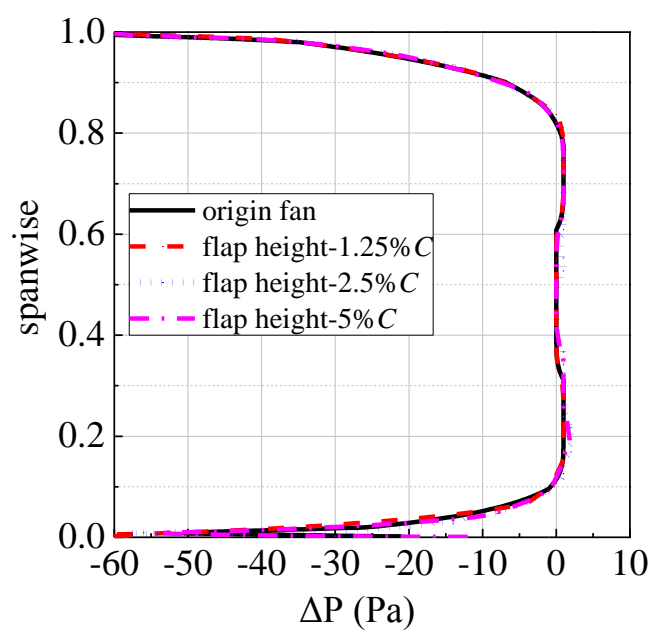

(a) $0 \%$ axial chord

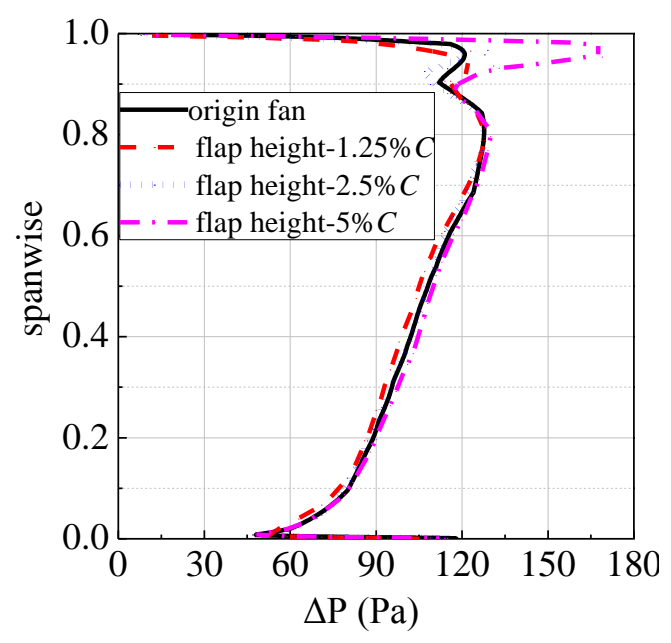

(c) $40 \%$ axial chord

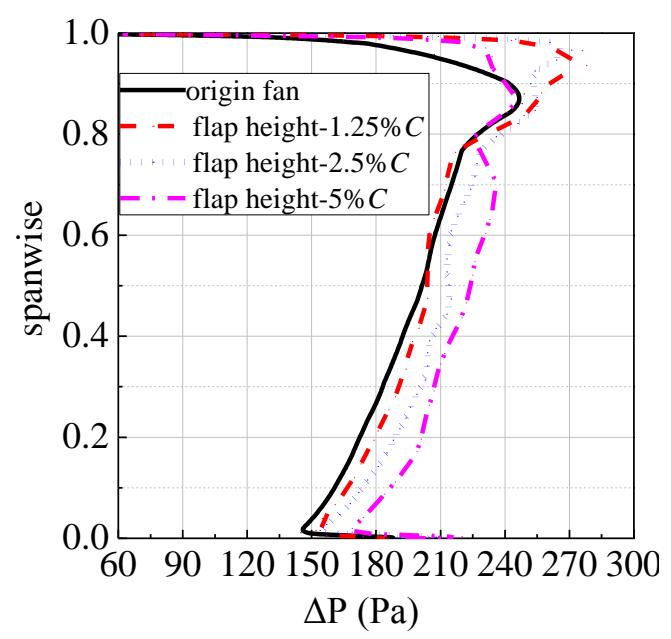

(e ) $80 \%$ axial chord

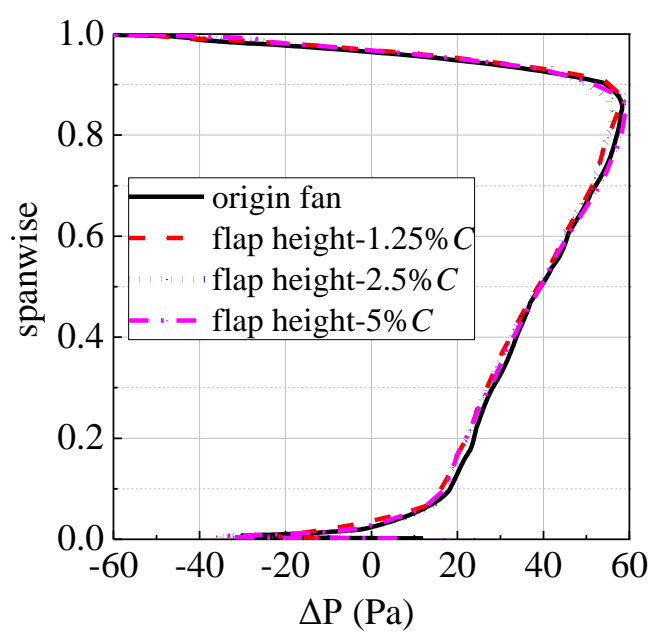

(b) $20 \%$ axial chord

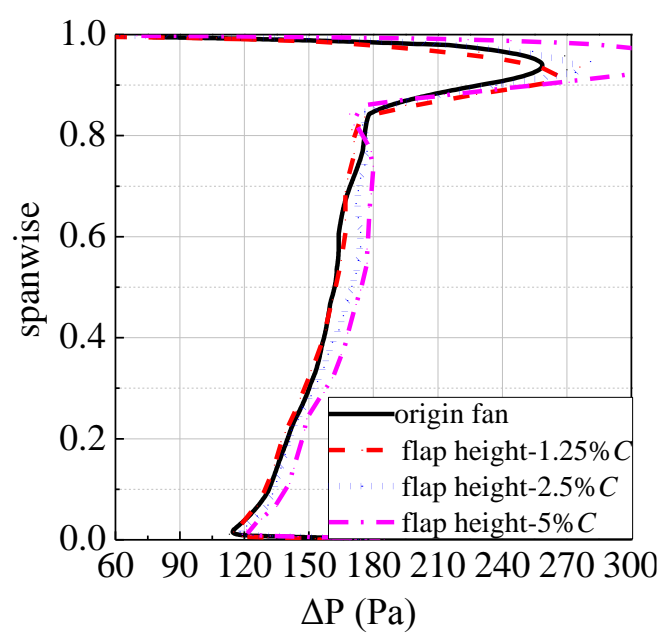

(d) $60 \%$ axial chord

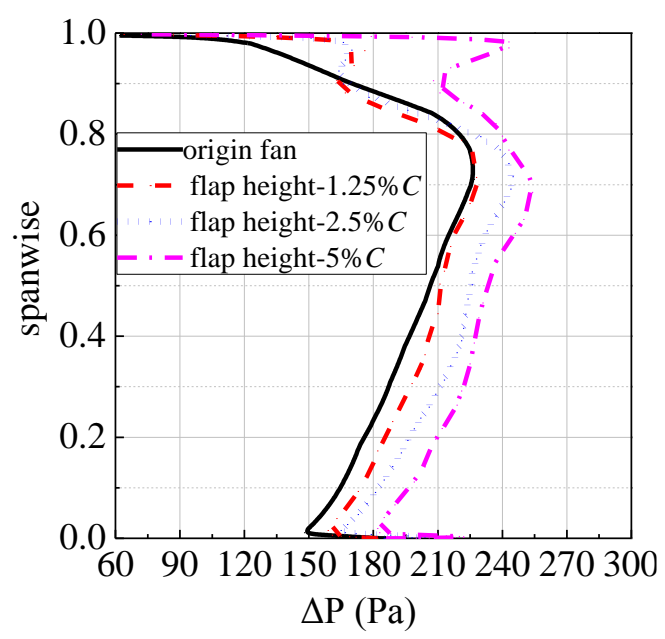

(f) $100 \%$ axial chord

Figure 15 Comparison of the total pressure rise at different axial positions with various GF 
heights.

419 Figure 16 shows the streamlines inside the blade row of a fan with a GF. Because the flap produces

420 an increased blade pressure difference between the pressure surface and suction surface, one

421 leakage flow is driven through the tip clearance from the pressure side to the suction side in the

422 form of a leakage vortex. From the previous section, it is found that a fan with a GF increases the

423 intensity of the trailing edge vortex, produces a high-pressure vortex zone in front of the flap,

424 accelerates the fluid flow on the suction surface and reduces the static pressure on the rear suction

425 surface. This is the reason for the increase in loss and decrease in efficiency of a fan with a GF. It is

426 also demonstrated by the flow pattern visualized in Figure 17.

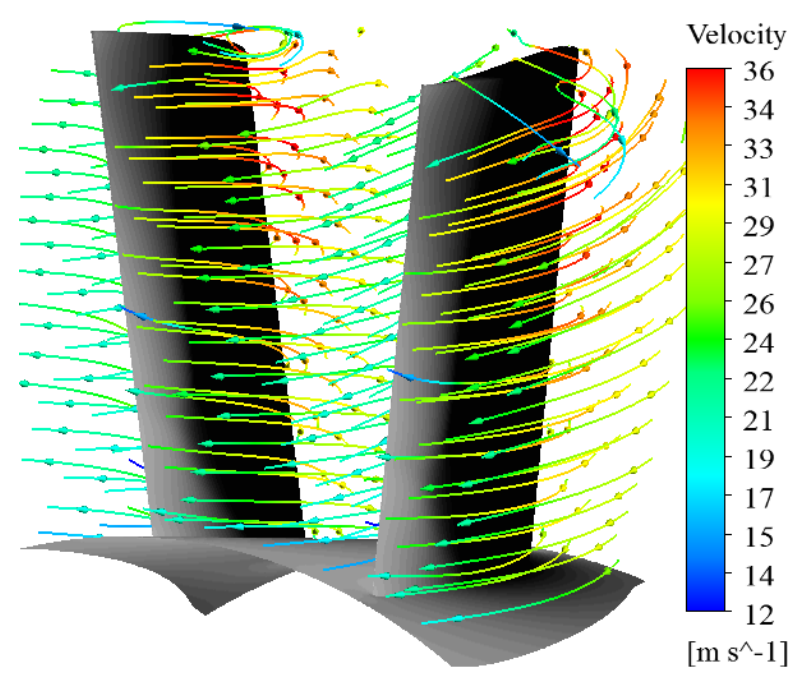

Figure 16 Streamlines inside the blade row of a flapped fan

427 Figure 17 presents the turbulent kinetic energy distribution on the cross-flow section at the trailing

428 edge of the blade. The turbulent kinetic energy is clearly increased at the outlet area by adding a

429 GF, especially in the area of the blade tip. The location of the tip clearance vortex is changed, moving toward the pressure surface. These colored contours more intuitively demonstrate the conclusions that the static pressure of the suction surface of the blade is decreased and the static pressure of the pressure surface is increased when a GF is installed. 


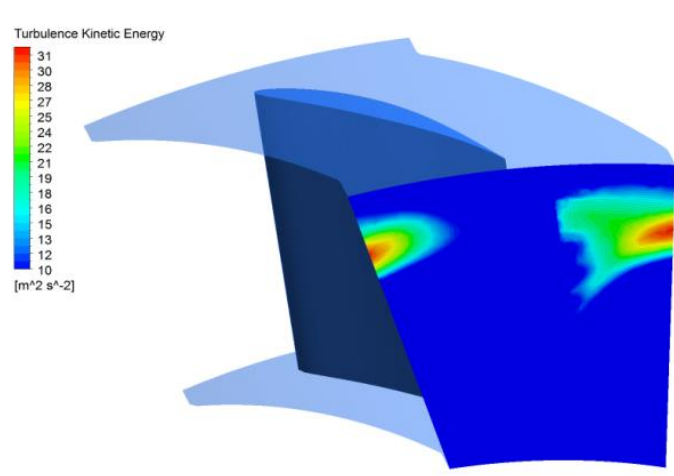

(a) Original fan

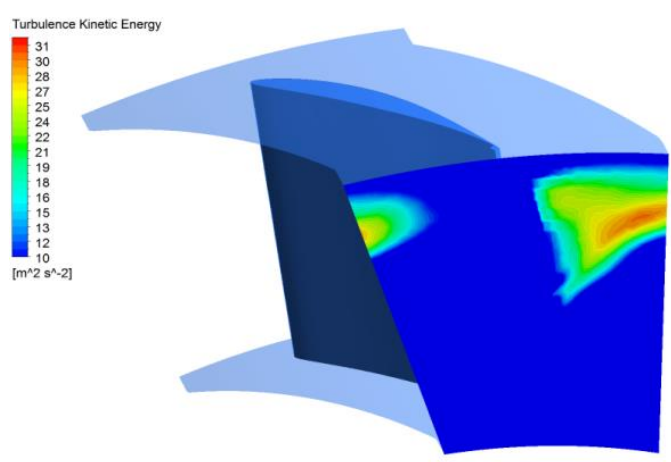

(c) GF of $2.5 \% \mathrm{C}$ height

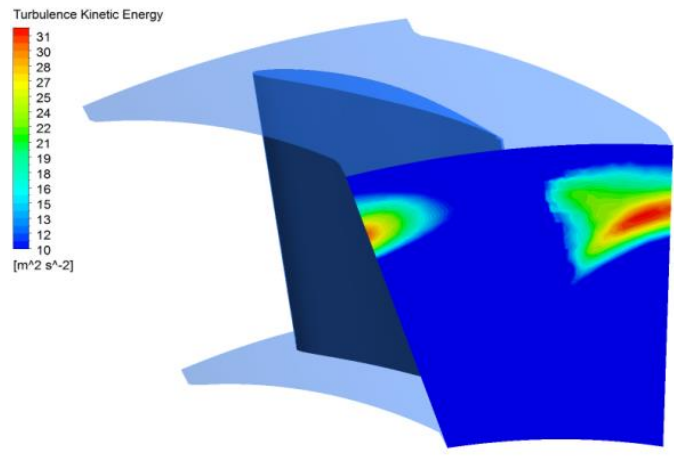

(b) GF of $1.25 \% \mathrm{C}$ height

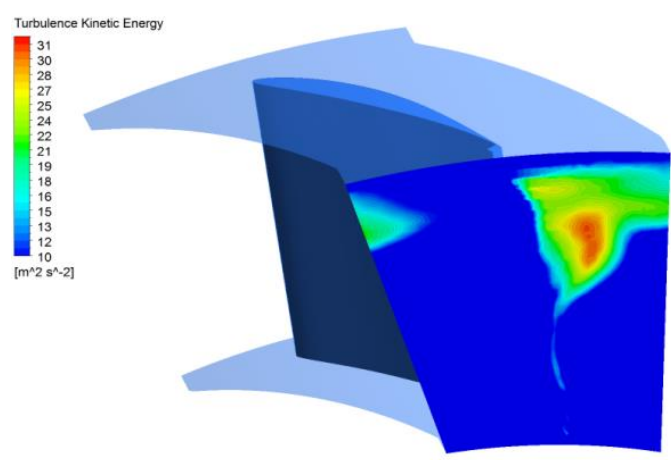

(d) GF of $5 \%$ C height

Figure 17 Turbulent kinetic energy distribution at the exit plane of the fan

\section{Conclusions}

This paper presents a computational and experimental investigation of the effect of the Gurney and $5 \%$ chord GFs. The following conclusions are drawn: the magnitude of the increase is enlarged with increase of GF's height. By contrast, the total pressure and static efficiency of the fan are reduced by GFs within the entire range of flow coefficients. However, the static pressure efficiency of a fan with a GF is increased compared with 
441 the original fan when the flow coefficient is $20 \%$ larger than the design value.

442 2. The computed pressure rise of the original fan and that of a fan with a $1.25 \%$ chord GF are 443 compared with the experimental data. The presented computational results agree reasonably well

444 with the available experimental data. This verifies that the CFD strategies chosen are reliable.

445 3. Through a numerical investigation, it is found that a fan with a GF can accelerate the flow and reduce the adverse pressure gradient in the blade row, which suppresses the flow separation at

447 the suction surface. The exit flow of the fan tends to be made axial by the GF. This indicates that a

448 fan with a GF produces a larger airflow-turning angle. Shedding vortices with high turbulence

449 intensities are clearly visible in the GF wake. An additional vortex region is created in front of the GF. These vortices are responsible for the increased suction on the upper surface and increased

451 pressure on the low surface. All of the above phenomena result in an increasing work capability of 452 the fan. The tip leakage vortex is strengthened by the increased suction on the suction surface, and 453 the increased pressure on the pressure surface is the main reason for the decrease in efficiency.

\section{Acknowledgment}

455 The work described in this paper was supported by the Chinese National Natural Science Funds 456 (No. 51276116).

\section{Nomenclature}

$\begin{array}{ll}D_{\mathrm{t}} & \text { diameter of the shroud } \\ D_{\mathrm{h}} & \text { diameter of the hub } \\ C & \text { chord length }\end{array}$




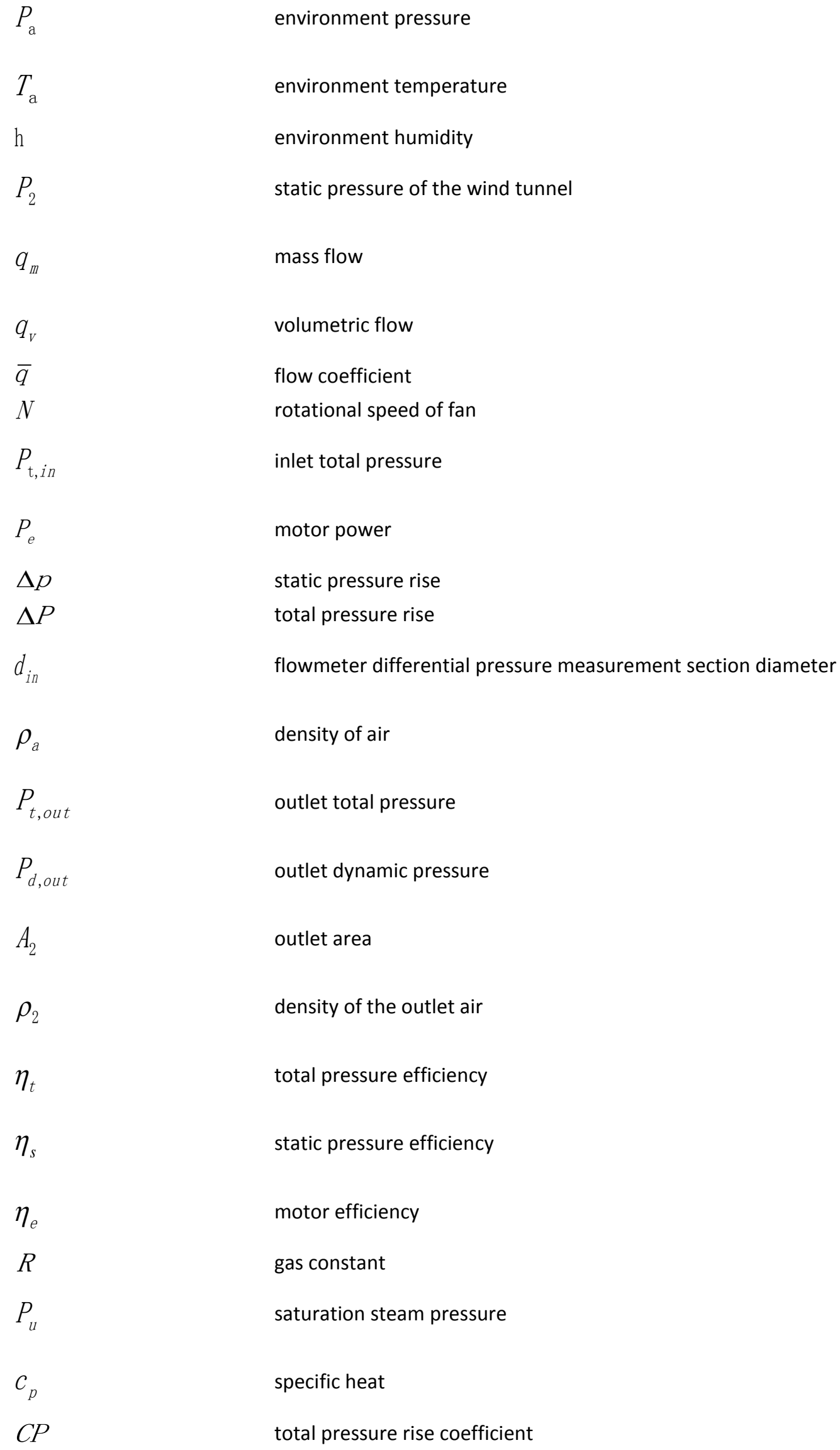




$\begin{array}{ll}C_{p} & \text { static pressure rise coefficient } \\ P_{6} & \text { Inlet total pressure of the test fan } \\ \Delta p^{\prime} & \text { static pressure rise of the flow nozzle } \\ \bar{p} & \text { static pressure coefficient }\end{array}$

\section{$458 \quad 8$ References}

459 1. Liebeck, R.H., Design of Subsonic Airfoils for High Lift. Journal of Aircraft, 1978. 15(9): p. 547$460 \quad 561$.

461

462

463

464

465

466

467

468

469

470

471

472

473

474

2. Wang, J.J., Y.C. Li, and K.S. Choi, Gurney flap-Lift enhancement, mechanisms and applications. Progress in Aerospace Sciences, 2008. 44(1): p. 22-47.

3. Wang, J., Y. Li, and P. Zhang, Influences of Mounting Angles and Locations on the Effects of Gurney Flaps. Journal of Aircraft, 2003. 40(3): p. 494-498.

4. Traub, L.W., A. Miller, and O. Rediniotis, Preliminary Parametric Study of Gurney Flap Dependencies. Journal of Aircraft, 2006. 43(43): p. 1242-1244.

5. Kobayashi, T., M. Takagi, and Y. Takkakura. Effects of Gurney Flaps for Aerodynamic Characteristics of an Airfoil. in NCTAM papers, National Congress of Theoretical and Applied Mechanics, Japan. 2012.

6. Dam, C.P.V., D.T. Yen, and P.M.H.W. Vijgen, Gurney Flap Experiments on Airfoil and Wings. Journal of Aircraft, 1999. 36(2): p. 484-486.

7. Dan, ,H., Pendergraft, , and C. Odis, A water tunnel study of Gurney flaps. 1988, NASA

8. Storms, B.L. and C.S. Jang, Lift enhancement of an airfoil using a Gurney flap and vortex generators. Journal of Aircraft, 1993. 31(3): p. 542-547. 
475 9. Ross, J.C., B.L. Storms, and P.G. Carrannanto, Lift-enhancing tabs on multielement airfoils. 476 Journal of Aircraft, 1995. 32(3): p. 649--655.

477 10. Myose, R., M. Papadakis, and I. Heron, Gurney Flap Experiments on Airfoils, Wings, and 478 Reflection Plane Model. Journal of Aircraft, 2012. 35(35): p. 206-211.

479 11. Papadakis, M., R. Myose, I. Heron, and B. Johnson. An experimental investigation of Gurney 480 flaps on a GA(W)-2 airfoil with 25 percent slotted flap. in Applied Aerodynamics Conference. 1996.

481 12. Ashby, D.L., Experimental and Computational Investigation of Lift-Enhancing Tabs on a Multi-

483

\section{Element Airfoil. 1997.}

13. Traub, L.W. and S.F. Galls, Effects of Leading- and Trailing-Edge Gurney Flaps on a Delta Wing. Journal of Aircraft, 1999. 36(4): p. 651-658.

14. Li, Y.C., J.J. Wang, G.K. Tan, and P.F. Zhang, Effects of Gurney flaps on the lift enhancement of a cropped nonslender delta wing. Experiments in Fluids, 2002. 32(1): p. 99-105.

15. Zhan, J.X. and J.J. Wang, Experimental Study on Gurney Flap and Apex Flap on Delta Wing. Journal of Aircraft, 2004. 41(6): p. 1379-1383.

16. Albertani, R., Wind-Tunnel Study of Gurney Flaps Applied to Micro Aerial Vehicle Wing. Aiaa Journal, 2008. 46(6): p. 1560-1562.

17. Cavanaugh, M., P. Robertson, and W. Mason, Wind Tunnel Test of Gurney Flaps and T-Strips on an NACA 23012 Wing. Aiaa Journal, 2006.

18. Buchholz, M.D. and T. Jin, Lift Augmentation on Delta Wing with Leading-Edge Fences and Gurney Flap. Journal of Aircraft, 2000. 37(6): p. 1050-1057.

19. J.M., J., Analysis of Industrial Design with Gurney Flaps, in 38th Aerospace Sciences Meeting 
496

497

498

499

500

501

502

503

504

505

506

507

508

509

510

511

512

513

\& Exhibit. 2000, AIAA paper: Reno. NV.

20. Byerley, A.R., O. StöRmer, J.W. Baughn, T.W. Simon, K.W.V. Treuren, and J.R. List, Using Gurney Flaps To Control Laminar Separation On Linear Cascade Blades. Journal of Turbomachinery, 2002. 125(1): p. 1191-1199.

21. Greenblatt, D., Application of Large Gurney Flaps on Low Reynolds Number Fan Blades. Journal of Fluids Engineering, 2011. 133(2): p. 352-361.

22. Thomas, M.K.D., N. Sitaram, and M. Suresh, Application of Gurney Flaps on a Centrifugal Fan Impeller. International Journal of Fluid Machinery \& Systems, 2012. 5(2): p. 65-71.

23. Vijgen, P.M.H.W., C.P.V. Dam, B.J. Holmes, and F.G. Howard, Wind-Tunnel Investigations of Wings with Serrated Sharp Trailing Edges. 1989: Springer Berlin Heidelberg. 295-313.

24. Zhang, L., Y. Jin, H. Dou, and Y. Jin, Numerical and Experimental Investigation on Aerodynamic Performance of Small Axial Flow Fan with Hollow Blade Root. Journal of Thermal Science, 2013. 22(5): p. 424-432.

25. Ye, X., P. Li, C. Li, and X. Ding, Numerical investigation of blade tip grooving effect on performance and dynamics of an axial flow fan. Energy, 2015. 82: p. 556-569.

26. Kim, J.H., J.H. Kim, K.Y. Kim, J.Y. Yoon, S.H. Yang, and Y.S. Choi, High-efficiency design of a tunnel ventilation jet fan through numerical optimization techniques. Journal of Mechanical Science \& Technology, 2012. 26(6): p. 1793-1800.

27. Kim, J.H., J.H. Choi, A. Husain, and K.Y. Kim, Performance enhancement of axial fan blade through multi-objective optimization techniques. Journal of Mechanical Science \& Technology, 2010. 24(10): p. 2059-2066. 
517 28. Singh, M.K., K. Dhanalakshmi, and S.K. Chakrabartty, Navier-Stokes Analysis of Airfoils with 518 Gurney Flap. Journal of Aircraft, 1971. 44(5): p. 1487-1493.

519 29. Jain, S., N. Sitaram, and S. Krishnaswamy, Computational Investigations on the Effects of

520 Gurney Flap on Airfoil Aerodynamics. International Scholarly Research Notices, 2015. 2015(5): p.

$521 \quad 1-11$

522

523 